DOI: $10.1002 / \operatorname{marc} .201500047$

\title{
Review
}

\section{Charge Carrier Transport and Photogeneration in P3HT:PCBM Photovoltaic Blends}

Frédéric Laquai, Denis Andrienko, Ralf Mauer, Paul W. M. Blom*

Assoc. Prof. F. Laquai, Dr. D. Andrienko, Dr. R. Mauer, Prof. P.W.M. Blom

Max Planck Institute for Polymer Research

Ackermannweg 10, D-55122 Mainz, Germany

E-mail: blom@mpip-mainz.mpg.de

Dr. R. Mauer

present address: InnovationLab GmbH, Speyerer Straße 4, 69115 Heidelberg / Germany

Assoc. Prof. F. Laquai

present address: Physical Sciences and Engineering Division (PSE), Material Science and Engineering (MSE), Solar and Photovoltaics Engineering Research Center (SPERC), King Abdullah University of Science and Technology (KAUST), Thuwal 23955-6900, Kingdom of Saudi Arabia

We review the charge transport and photogeneration in bulk-heterojunction solar cells made from blend films of regioregular poly(3-hexylthiophene) (RR-P3HT) and methanofullerene (PCBM). The charge transport, specifically the hole mobility in the RRP3HT phase of the polymer:fullerene photovoltaic blend, is dramatically affected by thermal annealing. The hole mobility increases more than three orders of magnitude and reaches a value of up to $2 \times 10^{-4} \mathrm{~cm}^{2} \mathrm{~V}^{-1} \mathrm{~s}^{-1}$ after the thermal annealing process, as a result of an improved semi-crystallinity of the film. This significant increase of the hole mobility balances the electron and hole mobilities in a photovoltaic blend in turn reducing spacecharge formation and it is the most important factor for the strong enhancement of the photovoltaic efficiency compared to an as cast, that is, non-annealed device. In fact, the balanced charge carrier mobility in RR-P3HT:PCBM blends in combination with a field- 
and temperature independent charge carrier generation and greatly reduced non-geminate recombination explains the large quantum efficiencies measured in P3HT:PCBM photovoltaic devices.

FIGURE FOR ToC_ABSTRACT

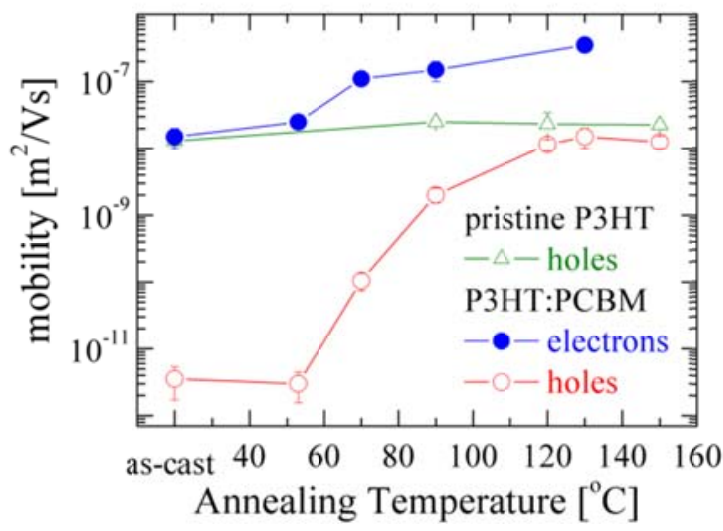




\section{Introduction}

The charge carrier mobility is one of the key parameters determining the efficiency of organic electronic devices such as light-emitting diodes (OLEDs), solar cells (OSCs) and field-effect transistors (OFETs). The mobility $\mu$ defines the drift velocity $v$ that a charge carrier obtains under the influence of an electric field $E$, that is, $v=\mu E$. The mobility is therefore a measure of how fast a charge carrier can travel in a semiconductor. Inorganic semiconductors are highly ordered materials with a periodic lattice leading to a delocalisation of charge carriers. The mean free path of charge carriers in these materials is high, and only limited by scattering of carriers with phonons. As a result, the charge carrier mobility in inorganic semiconductors decreases with increasing temperature. In contrast, the charge carrier mobility in organic semiconductors is observed to be strongly thermally activated. This behaviour is known from impurity conduction in inorganic semiconductors ${ }^{[1-3]}$ and from molecularly doped polymers, in which conjugated organic molecules are dispersed in an insulating polymer matrix. This latter class of materials was heavily investigated in the 60's and 70's due to their applicability in photocopying machines. In these materials charge transport takes place via 'hopping' between localized states. Although semiconducting polymers feature a delocalisation of charges, this delocalisation extends only in one dimension along the conjugated polymer backbone. Additionally, the conjugation is disrupted in many places due to torsion, impurities, chemical defects, and kinks in the polymer backbone. What results is a structure consisting of conjugated polymer segments of varying length on which charges are localized and need to 'hop' to other conjugated segments, similar as in molecularly doped polymers. This picture is in principle equivalent to an ensemble of oligomers of different length and as a consequence of the hopping process, the mobility in organic semiconductors is typically orders of magnitude lower than in inorganic semiconductors. Furthermore, the localised states in organic semiconductors are subject to spatial and energetic disorder. Carrier jumps upwards in energy may be thermally assisted by phonons to overcome the energy difference, leading to a 
strong temperature dependence of the mobility. The resulting mobility that is measured with, for instance, single-carrier devices, thus originates from the aggregate of many microscopic hopping events. The intrinsic nature of hopping transport is also the reason why the description of charge transport in molecularly-doped polymers is applicable to both, semiconducting polymers and small molecule devices, despite their dissimilar molecular structure.

From time-of-flight (TOF) studies and single-carrier SCL measurements, it has been deduced that the charge carrier mobility increases with electric field. ${ }^{[4,5]}$ The dependence of the mobility on electric field was found to have the form of a stretched exponential, as described by a Poole-Frenkel relation. ${ }^{[6]}$ The temperature dependence was observed to obey an Arrhenius-type temperature dependence. Combined with a Poole-Frenkel dependence this led to the following empirical equation for the charge carrier mobility.

$\mu(T, E)=\mu_{0}^{*} \exp \left[-\frac{\Delta}{k_{B} T}\right] \exp [\gamma \sqrt{E}]$

with $\mu_{0}{ }^{*}$ a mobility prefactor, $\Delta$ the activation energy, $k_{\mathrm{B}}$ Boltzmann's constant, $T$ the absolute temperature, $E$ the electric field, and $\gamma$ the field activation parameter. Note that the mobility prefactor $\mu_{0}{ }^{*}$ is equal to the mobility $\mu(E=0, T \rightarrow \infty)$ at zero electric field and in the limit of infinite temperature. Equation (1) has been used to describe charge transport in a variety of molecularly-doped polymers, however, it lacks proper theoretical justification. The origin of the stretched exponential term in the Poole-Frenkel theory lies in the description of how a coulomb potential near a charged localized state is modified by an electric field. In order to explain the charge transport in organic semiconductors in terms of the Poole-Frenkel theory it is required that every hopping site is equivalent to a trap that is neutral when filled by a charge carrier and electrically charged after its release, which would lead to unrealistically high trap densities. 
As stated above, specifically in conjugated polymers, but also in most organic semiconductors, charges are localized due to the large energetic disorder. Although the charge is delocalized over a certain conjugated segment, these segments are limited in size. Hence, the movement of charges in the material is limited by jumps from one segment to the other. This type of charge transport is typically described by hopping: phonon-assisted tunneling from transport site to site. By absorbing a phonon, a charge carrier can gain sufficient energy to hop. Consequently, the carrier mobility increases with increasing temperature. Many hopping models are based on the hopping rate proposed by Miller and Abrahams.[3] In their model the rate of charge carrier hopping from site $i$ to an unoccupied site $j$, that is $W_{i j}$, depends on the spatial distance, $R_{i j}$, and the energetic difference between the sites, $\Delta \varepsilon=\varepsilon_{j}-\varepsilon_{i}$, and was calculated as:

$$
W_{i j}=v_{0} \exp \left(-2 \alpha R_{i j}\right) \begin{cases}\exp \left(-\frac{\Delta \varepsilon}{k_{B} T}\right) & \Delta \varepsilon>0 \\ 1 & \Delta \varepsilon \leq 0\end{cases}
$$

where $v_{0}$ is the attempt-to-jump frequency, $\alpha^{-1}$ is an effective overlap parameter, $k_{B}$ is Boltzmann's constant, and $T$ is the absolute temperature. The exponential term on the left represents the tunneling probability, resulting in an exponential decrease of the rate with hopping distance. The exponential term on the right accounts for the phonon density. If the unoccupied site is lower in energy, then this term is unity, otherwise the hop is thermally assisted. In the pioneering work of Bässler, a charge transport model for disordered organic systems was proposed, based on hopping in a system with spatial and energetic disorder. The hopping rate was assumed to follow equation 2. In order to account for the statistical broadening of the HOMO and LUMO levels due to disorder a Gaussian density of states 
(DOS) was assumed. The approximation of a Gaussian DOS is, however, not arbitrary. In fact, it is supported by the observation of Gaussian-shaped optical spectra of electronic transitions. The transport in such a system cannot be solved analytically, and therefore Monte Carlo simulations were employed. The transition rates between hopping sites were given by the Miller-Abrahams model. ${ }^{[3]}$ Furthermore, it was assumed that not only the energy levels of the hopping sites, that are the HOMO for hole transport and LUMO for electron transport, exhibit a Gaussian distribution in energy, namely energetic or diagonal disorder, but that also the distance or the orientation between hopping sites exhibit a spreading, the so-called offdiagonal disorder. The mobility dependence proposed based on these assumptions and MonteCarlo calculations is now widely known as the Gaussian disorder model (GDM). ${ }^{[7]}$

$$
\mu_{G D M}(T, E)=\mu_{0}^{*} \exp \left[-\left(\frac{2}{3} \hat{\sigma}\right)^{2}+C\left(\hat{\sigma}^{2}-\Sigma^{2}\right) \sqrt{E}\right]
$$

with $\hat{\sigma} \equiv \sigma / k_{B} T$, in which $\sigma$ denotes the energetic disorder, $\Sigma$ a parameter describing the offdiagonal disorder, and $C$ an empirical constant depending on the distance between the hopping sites. The GDM exhibits a $\ln (\mu) \propto 1 / T^{2}$ dependence which is in contrast to Equation (1). However, in the temperature range that is typically measured the difference between a $1 / T$ and a $1 / T^{2}$ dependence is difficult to discriminate experimentally. Within the GDM, the field dependence according to Equation (1) is only reproduced in a limited electric field range, while such field dependence is experimentally observed over a much wider range of electric fields. An improvement of this model was made by taking into account spatial correlation between the energies of neighbouring sites. For example, the presence of dipoles in an organic semiconductor affects the energy landscape that a charge carrier experiences. As a result the energy of a hopping site is not fully random within the Gaussian distribution anymore, but it is correlated with the energy of neighbouring sites. This resulted in the so-called correlated 
disorder model (CDM). In this model the stretched exponential field dependence is extended to lower electric fields. ${ }^{[8-10]}$

$\mu_{C D M}(T, E)=\mu_{0}^{*} \exp \left[-\left(\frac{3}{5} \hat{\sigma}\right)^{2}+0.78\left(\hat{\sigma}^{3 / 2}-2\right) \sqrt{\frac{q a E}{\sigma}}\right]$

with $q$ the elementary charge and $a$ the distance between the hopping sites.

While the dependence of the mobility on electric field has been investigated intensively, it was realised much later that an additional factor influences mobility: the charge carrier density. The carrier density dependence of the mobility was difficult to determine from diode measurements, since in diodes an increase of the electric field is simultaneously accompanied by an increase of the charge carrier density. The key in disentangling the density- and electric field dependence of the mobility was the unification of diode and fieldeffect transistor (FET) measurements. In FETs the electric field between source and drain is much smaller than that in diodes, while the charge carrier density in the conductive channel is considerably larger than typically achieved in diodes. ${ }^{[11]}$ The measured mobilities in FETs are orders of magnitude larger than those in diodes. Additionally, a dependence of the mobility on the gate voltage is generally observed. ${ }^{[12,13]}$ Since the charge carrier density in the channel is controlled by the gate voltage, the gate-voltage dependence of the mobility can be explained by a charge carrier density dependence. ${ }^{[12,14,15]}$ The explanation for the charge carrier dependent mobility is deep state filling. Within the framework of the hopping model, charge transport is thought to take place in the tail of the DOS, in which only few hopping sites are available when the charge carrier density is low. As the density of charge carriers increases, more states become accessible by hopping, which in turn is reflected in a higher mobility.

Vissenberg and Matters obtained an analytical expression for the density dependence of the mobility based on variable range hopping in an exponential DOS. The resulting density dependence has the form of a power law according to ${ }^{[16]}$ 
$\mu(p)=\frac{\sigma_{0}}{q}\left(\frac{\left(T_{0} / T\right)^{4} \sin \left(\pi T / T_{0}\right)}{(2 \alpha)^{3} B_{c}}\right)^{T_{0} / T} p^{T_{0} / T-1}$

where $\sigma_{0}$ is a prefactor for the conductivity, $\alpha^{-1}$ is the effective overlap parameter between localized states, $B_{\mathrm{c}}$ the critical number for the onset of percolation that is equal to 2.8 for $3 \mathrm{D}$ systems and $T_{0}$ the characteristic temperature describing the decay of the exponential distribution. Taking into account a density dependent mobility, it could be shown that the difference between the mobilities in diodes and FETs is the direct result of the difference in the typical charge carrier densities. ${ }^{[17]}$ Moreover, it was demonstrated that at room temperature, the dependence of the mobility on the charge carrier density is dominant over the field dependence of the mobility. ${ }^{[18]}$ By acknowledging that the mobility depends on both electric field and charge carrier density, it also became clear that the mobility is not entirely a material property, but to some extent also dependent on the device architecture and measurement conditions.

Only recently, a full description of the mobility taking into account both the field and charge carrier density dependence was obtained by Pasveer et al. in the form of the extended Gaussian disorder model (EGDM). ${ }^{[19]}$ In this expression the dependencies of the mobility on electric field and charge carrier density are factored in field- and density enhancement functions.

$$
\begin{aligned}
& \mu_{E G D M}(T, p, E)=\mu_{0}^{*} c_{1} \exp \left[-c_{2} \hat{\sigma}^{2}\right] f(T, E) g(T, p) \\
& f(T, E)=\exp \left[0.44\left(\hat{\sigma}^{3 / 2}-2.2\right)\left(\sqrt{1+0.8\left(\frac{q a E}{\sigma}\right)^{2}}-1\right)\right] \\
& g(T, p)=\exp \left[\frac{1}{2}\left(\hat{\sigma}^{2}-\sigma\right)\left(2 p a^{3}\right)^{\delta}\right] \\
& \delta=2 \frac{\ln \left(\hat{\sigma}^{2}-\hat{\sigma}\right)-\ln (\ln (4))}{\hat{\sigma}^{2}}
\end{aligned}
$$


with $c_{1}=1.8 \times 10^{-9}$ and $c_{2}=0.42$. It should be noted that both mobility models describing the density dependence of the mobility assume a different shape of the DOS. The VissenbergMatters equation was obtained for an exponential DOS, while the EGDM is based on a Gaussian DOS. The fundamental difference between an exponential DOS and a Gaussian DOS is, that while in the former system the average energy of the hopping carriers relaxes to $-\infty$, it relaxes to $-\sigma^{2} / k_{\mathrm{B}} T$ in the latter. During a voltage sweep only a small part of the DOS is filled. Consequently, the segment of a Gaussian that is being filled during a voltage sweep can be approximated by an exponential. However, the reverse can also be true. There are only a few reports on experimental measurements of the shape of the DOS in organic semiconductors. Hulea et al. measured a complex DOS in PPV consisting of a Gaussian core, and a tail that contains both, features of a Gaussian as well as an exponential distribution, while Tal et al. found an exponential tail for the DOS of the small organic molecule $\alpha$ NPD. ${ }^{[20]}$ Finally, from a computational study it was predicted that the tail of the DOS of poly(3-hexylthiophene) is exponential rather than Gaussian. ${ }^{[21]}$ The various mobility models used to describe the charge transport in organic semiconductors depend critically on the assumed shape of the DOS. The actual shape of the DOS is therefore a major source of uncertainty. 


\section{Charge Transport, Generation and Extraction}

\subsection{Charge Transport in Pristine P3HT and P3HT:PCBM Blends}

The charge carrier mobility of P3HT and its blends with fullerene derivatives depends heavily on several parameters such as the regioregularity of the polymer, its molecular weight, the processing conditions used for film preparation and any post-processing annealing procedures. Consequently, the photovoltaic device performance is also impacted by all of the aforementioned parameters. As a rule of thumb, high and balanced charge carrier mobilities are beneficial for device performance, for instance in photovoltaic devices non-geminate recombination of charges is suppressed by fast carrier extraction and a balanced carrier mobility reduces space-charge formation in the photoactive layer. The charge carrier mobility of pristine P3HT and its photovoltaic blends with fullerene as acceptor has been studied by several groups in the past.

In an early study Mihalietchi et al. ${ }^{[22]}$ investigated the charge transport and photogeneration in regioregular poly(3-hexylthiophene):methanofullerene (RRP3HT:PCBM) solar cells. Figure 1 shows the experimental $J_{\text {ph }}$ of such RR-P3HT:PCBM blends (50:50 wt.-\%) in a double logarithmic plot as a function of the effective applied voltage $\left(V_{0}-V\right) .{ }^{[23]}$ By correcting the applied voltage $V$ with the built-in voltage $V_{o}$ the effective voltage $\left(V_{0^{-}} V\right)$ represents the net electric field in the device. In this way the photocurrent can be visualized as a function of the electric field in the solar cell. The curves correspond to different postproduction treatments, specifically: as-cast, thermally annealed at a temperature where the enhancement in power conversion efficiency $\eta$ is maximized (120 ${ }^{\circ} \mathrm{C}$ ), and annealed at lower temperature $\left(70^{\circ} \mathrm{C}\right)$. Thermal annealing was performed on complete devices, that is, with the photoactive layer between the electrodes, on a hot plate for a period of 4 minutes. 


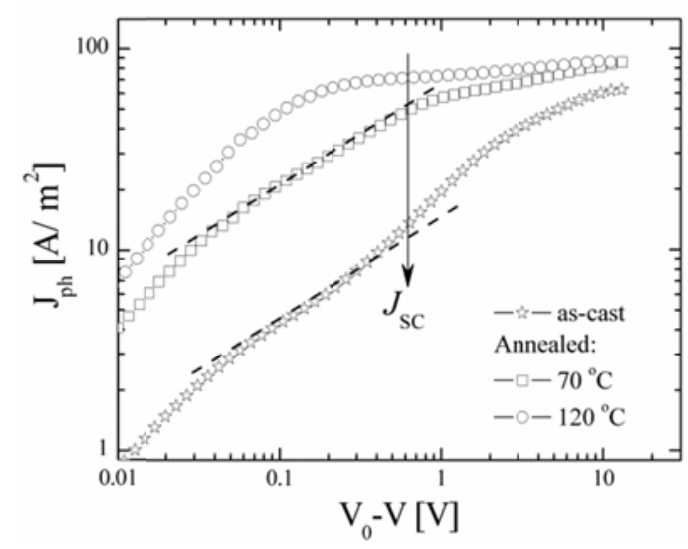

Figure 1. Experimental photocurrent $\left(J_{\mathrm{ph}}\right)$ versus effective applied voltage $\left(V_{0}-V\right)$ of P3HT:PCBM devices at room temperature for an as-cast device and after thermally annealing of the photoactive layers (see legend). The device thickness $(L)$ is $96 \mathrm{~nm}$ and the arrow indicates the position of the short-circuit current $\left(J_{\mathrm{SC}}\right)$. The dashed lines represent a squareroot dependence of $J_{\mathrm{ph}}$ on voltage. Reproduced from Adv. Funct. Mater. 2006, 16, 699-708.

Figure 1 shows that the photocurrent is strongly enhanced after thermal annealing. For the completely annealed device (at $120^{\circ} \mathrm{C}$ ) the short-circuit current $\left(J_{\mathrm{SC}}\right)$ increases by a factor of 5 , the fill factor (FF) by a factor of 2 and the overall enhancement of the power conversion efficiency is about an order of magnitude when compared to the as-cast device. It has been suggested that this dramatic boost in efficiency as a result of thermal treatment of the photoactive layer is caused by burning of shunts, ${ }^{[24]}$ an increase in hole mobility due to an improvement of semi-crystallinity of the polymer, changes in morphology, and an improved overlap with the solar emission due to a red shift of the optical absorption. ${ }^{[24-27]}$ The effect of the thermal annealing process on the solar cell performance was further quantified in terms of physical parameters, specifically the charge carrier mobility, charge generation and recombination in these devices.

For this analysis a device model was used that quantitatively describes the characteristics of organic bulk heterojunction solar cells. The dissociation efficiency of 
bound electron-hole (e-h) pairs, created after photoinduced electron transfer at the donoracceptor interface, is an important efficiency-limiting factor in photovoltaic devices. ${ }^{[28-30]}$ Furthermore, with regard to charge transport, it was also demonstrated that the photocurrent reaches the fundamental space-charge limit when the difference in electron and hole mobility exceeds two orders of magnitude. ${ }^{[31]}$ This model has been applied to understand the effect of postproduction annealing at different temperatures on the performance of P3HT:PCBM bulk heterojunction solar cells, and to quantify the parameters that limit the device performance.

Prior to the investigation of the photocurrent of P3HT:PCBM blends, knowledge about the hole and electron mobilities is indispensable. Much work has been done to measure the hole mobility of pristine P3HT using field-effect transistors (FET), ${ }^{[32,33]}$ time-of-flight (TOF) photocurrent measurements, ${ }^{[34]}$ and space-charge limited (SCL) current in a sandwich structure corresponding to solar cells or light-emitting diode configurations. ${ }^{[35]}$ The measured hole mobility ranges from $10^{-4} \mathrm{~cm}^{2} \mathrm{~V}^{-1} \mathrm{~s}^{-1}$ in TOF and SCL, and up to $10^{-1} \mathrm{~cm}^{2} \mathrm{~V}^{-1} \mathrm{~s}^{-1}$ in FETs. It should be noted that regioregular P3HT self-organizes into a semi-crystalline structure and due to the $\pi-\pi$ stacking direction the charge (hole) transport is extremely efficient. Since in FET measurements the current travels in the plane of the film, that is parallel to the substrate, the anisotropy in the polymer chain orientation strongly contributes to the difference of the measured mobilities. ${ }^{[36]}$ Moreover, a different molecular-weight of P3HT, the presence of PCBM and/or applying annealing also affects the measured electron and hole mobilities. ${ }^{[33,35]}$ Therefore, the relevant values for charge carrier mobilities can only be obtained when measured in the exact same configuration and experimental conditions as used in an operational solar cell device.

The electron and hole mobilities in the blend can be determined from current-voltage measurements by using suitable electrodes which either suppress the injection of electrons or holes, resulting in a hole- or electron-only device, respectively. ${ }^{[28,37]}$ A schematic diagram of a 
hole-only device is shown in the inset of Figure 2a, and a diagram of an electron-only device is presented as inset in Figure $\mathbf{2 b}$.

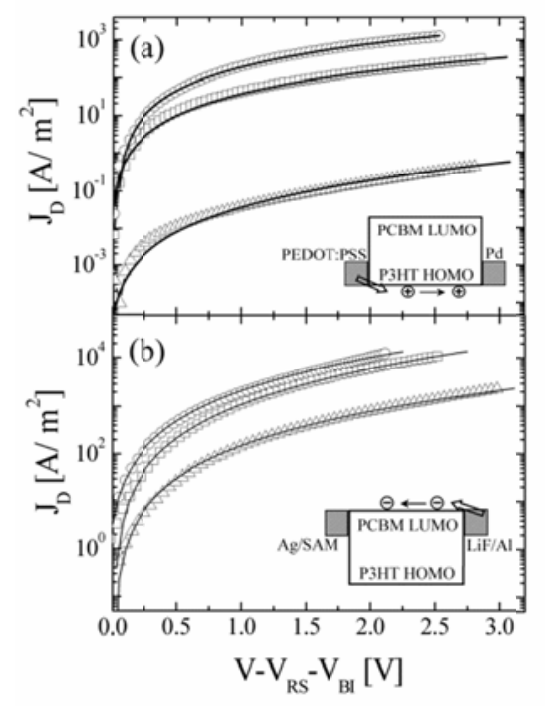

Figure 2. Experimental dark current densities $\left(J_{\mathrm{D}}\right)$ of a 50:50 wt.\% RR-P3HT:PCBM blend device measured at room temperature in the hole-only (a) and electron-only (b) device configuration, as shown by the inset schematic diagrams. The symbols correspond to different thermal annealing temperatures of the photoactive layer, that are, as-cast $(\Delta), 90{ }^{\circ} \mathrm{C}(\square)$, and $120{ }^{\circ} \mathrm{C}(\circ)$, respectively. Here, the thickness of the annealed hole-only devices $(\square, \circ)$ is 120 $\mathrm{nm}$, whereas for the other devices it is $220 \mathrm{~nm}$. The solid lines represent fits according to a model of single carrier SCL current with field-dependent mobility. The $J_{\mathrm{D}^{-}} V$ characteristics are corrected for the voltage drop over the contacts $V_{\mathrm{RS}}$ and the built-in voltage $V_{\mathrm{BI}}$ that arises from the work function difference between the contacts. Reproduced from Adv. Funct. Mater. 2006, 16, 699-708.

Figure 2 shows the experimental dark current densities $\left(J_{\mathrm{D}}\right)$ of P3HT:PCBM blends that were measured in hole-only (Figure 2a) and electron-only (Figure 2b) devices for different thermal annealing temperature performed on the completed device. For clarity, only the devices that were measured as-cast, after thermal annealing at $90{ }^{\circ} \mathrm{C}$ and $120{ }^{\circ} \mathrm{C}$, are 
shown. The applied voltage was corrected for the built-in voltage $\left(V_{\mathrm{BI}}\right)$ and the voltage drop $\left(V_{\mathrm{RS}}\right)$ due to the substrate's series resistance. When the applied voltage is greater than $V_{\mathrm{BI}}, J_{\mathrm{D}}$ for all devices increases quadratically with voltage, indicative of space-charge limited charge transport. This observation is typical for low mobility disordered semiconductors and it allows for a direct determination of the charge carrier mobility. ${ }^{[28,35,37]}$

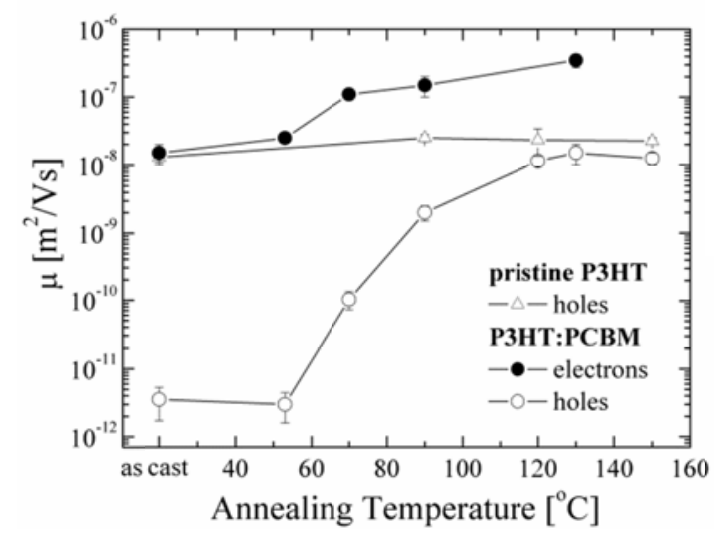

Figure 3. Room temperature electron $(\bullet)$ and hole (०) zero-field mobilities in 50:50 wt.-\% blends of RR-P3HT:PCBM as a function of postproduction annealing temperature of the completed devices. For comparison, the hole mobility measured in pristine P3HT devices $(\Delta)$ is also shown. The mobilities were calculated from the SCL currents measured in electronand hole-only device configurations (compare Figure 2). Reproduced from Adv. Funct. Mater. 2006, 16, 699-708.

Figure 3 shows the calculated zero-field mobility of electrons and holes in 50:50 wt.$\%$ blends of P3HT:PCBM devices as a function of the annealing temperature. For comparison, the hole mobility of pristine $\mathrm{P} 3 \mathrm{HT}$, measured under the same experimental conditions, is also shown. Figure 3 indicates that the hole mobility in pristine P3HT is hardly affected by thermal annealing, with a typical value of $(1.4-3.0) \times 10^{-4} \mathrm{~cm}^{2} /$ Vs. This mobility was found to be completely field independent and is fully consistent with previously reported values for high molecular-weight RR-P3HT (as the one used here). ${ }^{[35]}$ In contrast, the hole mobility of RR-P3HT in the blend is strongly affected by the presence of PCBM, in fact, it drops almost 4 orders of magnitude for an as-cast device. Upon annealing, however, the 
mobility starts to increase sharply at an onset of $50-60{ }^{\circ} \mathrm{C}$ followed by saturation at approximately the level of the pristine polymer, when the devices are annealed above $120{ }^{\circ} \mathrm{C}$. Moreover, the electron mobility of PCBM in the blend is also affected by thermal annealing: for as-cast films the electron mobility is $(1-2) \times 10^{-4} \mathrm{~cm}^{2} \mathrm{~V}^{-1} \mathrm{~s}^{-1}$, typically a factor of 5000 higher than the hole mobility. As a result, the charge transport in as-cast films is strongly unbalanced and the current is fully dominated by electrons. The experimental charge-carrier mobilities depicted in Figure 3 were used to analyze the photocurrent generation and the performance of the solar cells as a function of the thermal annealing temperature as will be discussed in a succeeding section.

Charge carrier mobilities similar to those reported by Mihailetchi et al. (vide supra) were also obtained by Yang et al. who investigated the electron and hole transport in pristine regioregular P3HT films. ${ }^{[38]}$ Carrier mobilities on the order of $4 \times 10^{-4} \mathrm{~cm}^{2} \mathrm{~V}^{-1} \mathrm{~s}^{-1}$ were found for electrons and holes, yet only dispersive transport was observed. The mobilities were found to be almost field-independent, however, the temperature dependence was not investigated in this study. In line with Mihailetchi et al., ${ }^{[22]}$ Huang et al. studied the effect of composition and annealing on the charge transport properties of $\mathrm{P} 3 \mathrm{HT}$ blends with $\mathrm{PC}_{61} \mathrm{BM}$, but now using the time-of-flight technique instead of SCLC ${ }^{[39]}$ They found a transition from dispersive to nondispersive transport upon increasing the fullerene fraction and balanced charge carrier mobilities at a weight ratio of $1: 1$. The mobility was found to be only weakly field-dependent with low-field electron and hole mobilities approaching $10^{-4} \mathrm{~cm}^{2} \mathrm{~V}^{-1} \mathrm{~s}^{-1}$. At higher PCBM loadings the transport became dispersive and only at a 1:1 ratio both electron as well as hole transport were found to be non-dispersive in part explaining the optimum photovoltaic performance for a 1:1 blending ratio. Unfortunately, the authors did not mention the molecular weight, polydispersity and regioregularity of the P3HT investigated in their study, which makes it difficult to directly compare their results with other studies. Likewise Baumann et al. investigated charge transport in P3HT:PCBM blends of varying composition 
by TOF experiments. ${ }^{[40]}$ They observed two transit times for electrons at P3HT:PCBM ratios of 1:4 and 1:1 and for hole transients at a ratio of 1:2 with hole and electron mobilities on the order of $10^{-4}-10^{-3} \mathrm{~cm}^{2} \mathrm{~V}^{-1} \mathrm{~s}^{-1}$ and ambipolar transport in pristine P3HT with a mobility in the range of $10^{-4} \mathrm{~cm}^{2} \mathrm{~V}^{-1} \mathrm{~s}^{-1}$. Mauer et al. presented a time-of-flight study on charge carrier transport in pristine $\mathrm{P} 3 \mathrm{HT}$ and its blends with $\mathrm{PCBM}$, specifically the carrier mobility in regiorandom P3HT (RRa-P3HT), regioregular P3HT (RR-P3HT) with $95 \%$ and $98 \%$ regioregularity and $\mathrm{RR}-\mathrm{P} 3 \mathrm{HT}$ blended with $\mathrm{PC}_{61} \mathrm{BM}$ as a function of the electric field and temperature. ${ }^{[41]}$ The experiments showed that hole transport in regiorandom P3HT (RRaP3HT) is largely dispersive. This is a straightforward consequence of the large disorder of the polymer introduced by torsion along the polymer backbone due to steric hindrance between adjacent alkyl-sidechains suppressing the pi-overlap between chains and thus limiting the semi- crystallinity and interchain transport. Furthermore, the glass transition temperature of $\mathrm{RRa}-\mathrm{P} 3 \mathrm{HT}$ is around room temperature and hence the polymer is not in a glassy amorphous state with significant side chain motion likely perturbing charge transport. In contrast, hole transport in regioregular $\mathrm{P} 3 \mathrm{HT}$ was found to be partially non-dispersive as the TOF photocurrent transients exhibited a short but plateau-like region. Figure 4 shows examples of respective time-of-flight hole transients of pristine RRa-P3HT and RR-P3HT films.

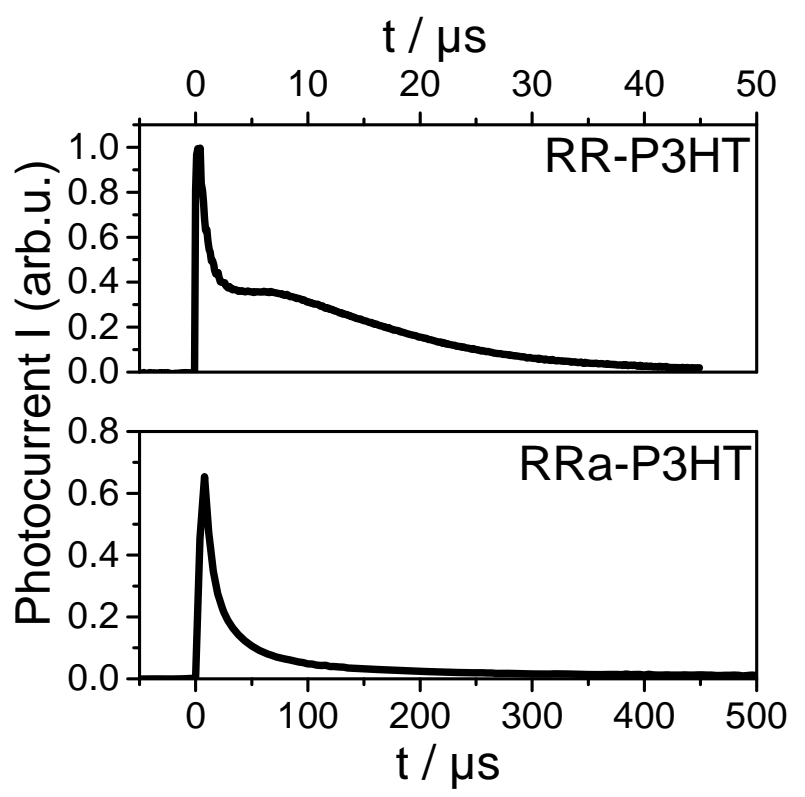


Figure 4. Representative time-of-flight hole transients of regioregular (98\%) RR-P3HT (top panel) and regiorandom RRa-P3HT. Note the different scaling of the time axis. Reproduced from data reported in Adv. Funct. Mater. 2010, 20, 2085.

The hole mobility was found to be higher for the polymer with higher regioregularity, most likely as a consequence of increased order and thus facilitated interchain transport. However, the two RR-P3HT polymers studied by Mauer et al. ${ }^{[41]}$ differed also in their molecular weight and polydispersity, which can have an additional impact on the hole mobility as demonstrated by Zen et al. ${ }^{[42]}$ The hole mobility in RR-P3HT was found to be virtually field-independent at moderate electric fields, but exhibited a moderate temperature dependence as shown in Figure 5.

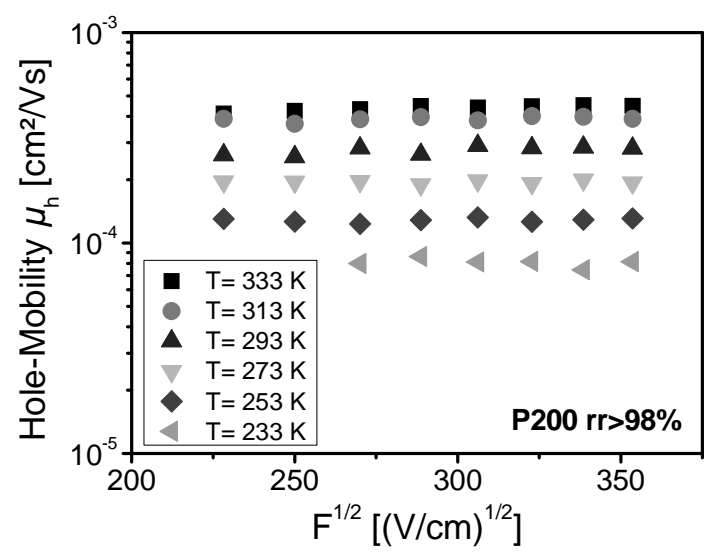

Figure 5. Semilogarithmic representation of the field- and temperature dependence of the hole mobility of regioregular ( $>98 \%$ ) RR-P3HT. Reproduced from Adv. Funct. Mater. 2010, $20,2085$.

This is characteristic for a material with low energetic disorder and no static dipoles. In fact, analyzing the temperature dependence of the mobility measurements in the framework of the Gaussian Disorder Model (GDM) as proposed by Bässler and coworkers revealed an energetic disorder parameter of only about $58 \mathrm{meV} .{ }^{[41]}$ This is significantly lower than found for many other conjugated polymers, which often exhibit energetic disorder parameters close to or even above $80-100 \mathrm{meV} \cdot{ }^{[43,44]}$ Cowan et al. performed transient photoconductivity studies on P3HT:PCBM solar cells and reported mobilities ranging from $4 \times 10^{-4} \mathrm{~cm}^{2} \mathrm{~V}^{-1} \mathrm{~s}^{-1}$ to $9 \times 10^{-4} \mathrm{~cm}^{2} \mathrm{~V}^{-1} \mathrm{~s}^{-1}$ for the slowest and fastest carriers, respectively. ${ }^{[45]}$ The authors demonstrated 
that in P3HT:PCBM and PCDTBT:PCBM, a carbazole-containing copolymer commonly used for organic photovoltaic devices, on the one hand a competition between sweep-out of carriers driven by the internal electric field itself limited by the charge carrier mobility and on the other hand a loss of photogenerated carriers by non-geminate recombination determines the bias dependence of the photocurrent. Overall, all charge carrier mobility studies performed on P3HT:PCBM demonstrated that the bulk hole and electron mobility is on the order of $10^{-4}$ $\mathrm{cm}^{2} \mathrm{~V}^{-1} \mathrm{~s}^{-1}$ with largely balanced mobilities at a mixing ratio of $1: 1$ and a weak fielddependence of the charge carrier mobility.

\subsection{Charge Carrier Generation in P3HT:PCBM}

The charge carrier generation rate is the measure taking into account all processes that ultimately lead to the generation of free charge carriers including absorption of photons, formation of singlet excitons, exciton diffusion and their dissociation at the heterojunction as well as the formation of coloumbically-bound charge-transfer (CT) states, their recombination and alleged dissociation. It is determined by the photon flux of incident solar illumination in the spectral range absorbed by the photoactive layer. For instance, the absorption onset of a prototypic annealed RR-P3HT:PCBM solar cell is typically around $\sim 620 \mathrm{~nm}$. Integrating the photon flux of the AM1.5G solar spectrum in the spectral range between $280 \mathrm{~nm}$ and $620 \mathrm{~nm}$, the latter being the absorption onset of P3HT:PCBM, yields $9.97 \times 10^{16}$ photons $\mathrm{cm}^{-2} \mathrm{~s}^{-1}$, resulting in an upper limit of the charge generation rate of $\mathrm{G}_{\max }=9.97 \times 10^{21} \mathrm{~cm}^{-3} \mathrm{~s}^{-1}$ in a photoactive layer of $\sim 100 \mathrm{~nm}$, however, assuming that all incident photons are absorbed in the photoactive layer. Clearly, this is not the case in common photoactive layers, as the thickness is typically limited to not more than a few hundred nanometers and as the absorption of organic molecules is intrinsically limited to certain spectral regions corresponding to the electronic transitions. This in turn causes a lower charge generation rate and several strategies have been developed to increase the absorbance of the device such as the fabrication of tandem solar cells consisting of sub-cells each optimized for a certain spectral region ${ }^{[46]}$ and 
the development of non-fullerene acceptors that unlike the fullerenes can contribute to the absorbance of the photoactive layer. ${ }^{[4]}$ Furthermore, photon losses due to reflection at interfaces, waveguiding in the active layer and parasitic absorption of the individual layers in the device reduce the performance. Optical simulations using a transfer matrix formalism presented by Burkhard et al. could give an estimate of the real absorption of the photoactive layer in a device by taking into account the aforementioned parasitic losses. ${ }^{[48]}$ It turned out that reflection off the back electrode and parasitic absorption by the metal electrodes reduces the charge generation rate by $10-50 \%$, depending on the thickness and optical constants of each layer in the device. Furthermore, interference effects can modulate the absorption in turn modulating the photocurrent and efficiency as a function of the photoactive layer thickness. ${ }^{[49]}$ Absorption measurements on P3HT:PCBM solar cells with 4\% power conversion efficiency have demonstrated that the parasitic losses account for a reduction of the charge generation rate by $\sim 20 \%$ yielding $\mathrm{G}_{\mathrm{abs}}=7.98 \times 10^{21} \mathrm{~cm}^{-3} \mathrm{~s}^{-1} .8$

After absorption of photons by the photoactive layer charge transfer occurs at the polymer:fullerene interface. There is compelling evidence that this process is largely ultrafast, that is, it occurs on a sub-100fs timescale, implying that photogenerated excitons do not have to diffuse prior to charge transfer at the interface. However, in recent transient absorption studies on annealed RR-P3HT:PCBM blends it was observed that phase segregation between P3HT and PCBM leads to a small diffusion-limited exciton dissociation component within the first $10 \mathrm{ps}$ after photoexcitation. ${ }^{[50,51]}$ This is rather fast compared to the exciton lifetime in pristine P3HT films of $(576 \pm 2)$ ps as determined by time-resolved photoluminescence spectroscopy, ${ }^{[41]}$ implying that less than $2 \%$ of the excitons recombine before reaching an interface and before being dissociated. Overall the impact of exciton recombination on the total exciton dissociation rate is low and including losses due to exciton recombination yields $\mathrm{G}_{\mathrm{exc}}=7.82 \times 10^{21} \mathrm{~cm}^{-3} \mathrm{~s}^{-1}$. 
The product of exciton quenching at the interface can be free charge carriers and coloumbically-bound interfacial charge-transfer states. The latter are created if charges do not manage to entirely escape their mutual attraction during the charge transfer process. Recent transient absorption (TA) experiments by Howard et al. suggested that in annealed blends of RR-P3HT and PCBM more than $80 \%$ of the excitons dissociate into free charge carriers and less than $20 \%$ create bound CT states. ${ }^{[51]}$ This is in stark contrast to blends of regiorandom (RRa) P3HT and PCBM in which the splitting ratio is reversed, yielding $20 \%$ free charge carriers and $80 \% \mathrm{CT}$ states. However, the driving force for electron transfer from P3HT to PCBM is very similar in these two systems and thus the origin of the substantial difference is likely associated with the different morphology of the blends. In RRa-P3HT:PCBM diffusionlimited exciton dissociation was not observed, implying that all photogenerated excitons dissociate in less than 100 fs. This in turn indicates a much smaller component demixing likely equivalent to molecular dispersion of PCBM molecules in a RRa-P3HT polymer matrix. However, a certain degree of phase segregation and fullerene aggregation appears to be required for efficient charge carrier separation, as previous studies have demonstrated. ${ }^{[52,53]}$ In line with these results as-cast RR-P3HT:PCBM blends, which exhibit an intermediate degree of demixing and reduced semi-crystallinity of both the P3HT and fullerene component and thus rank in between RRa-P3HT:PCBM and annealed RR-P3HT:PCBM blends, showed a free carrier yield of $68 \%$ versus $32 \%$ charge-transfer states. ${ }^{[51]}$ Burkhard et al. determined an internal quantum efficiency (IQE) of P3HT:PCBM solar cells of $80 \%$ (at short circuit conditions), ${ }^{[48]}$ which is in excellent agreement with the $20 \%$ loss by geminate recombination of CT-states demonstrated by TA experiments. ${ }^{[51]}$ Finally, the external quantum efficiency (EQE) of optimized P3HT:PCBM solar cells has frequently been reported to be $\sim 65 \%$, which is perfectly in line with a loss of $20 \%$ due to absorption and another $20 \%$ due to geminate recombination of CT-states in turn yielding a total loss of $36 \%$. Hence, approximating the charge generation rate in P3HT:PCBM including all of the aforementioned processes yields 
$\mathrm{G}_{\mathrm{SSC}}=6.26 \times 10^{21} \mathrm{~cm}^{-3} \mathrm{~s}^{-1}$, irrespective of the applied electric field and irrespective of the temperature. Assuming that all photogenerated free charge carriers can be extracted from the device prior to non-geminate recombination yields an upper limit of the photocurrent of 10.0 $\mathrm{mA} / \mathrm{cm}^{2}$, which is in good agreement with the short circuit current of optimized P3HT:PCBM solar cells that have a $\sim 4 \%$ power conversion efficiency.

\subsection{The effect of thermal annealing on solar cell performance}

After reviewing the charge transport and optical absorption properties of P3HT:PCBM solar cells we proceed with a more detailed discussion of the changes of the solar cell performance upon thermal annealing of the photoactive 50-50 wt.\% RR-P3HT:PCBM layer. Figure 6 shows the variation of the principal photovoltaic parameters that are their mean values together with the standard deviations, as a function of the annealing temperature. The devices were illuminated by a halogen lamp, intensity-calibrated by a silicon photodiode at an intensity of approximately $1.15 \mathrm{Sun}\left(115 \mathrm{~mW} / \mathrm{cm}^{2}\right)$. The $V_{\mathrm{OC}}$ was found to be relatively constant and varied less than $40 \mathrm{mV}$ with a slight increase above $150{ }^{\circ} \mathrm{C}$.

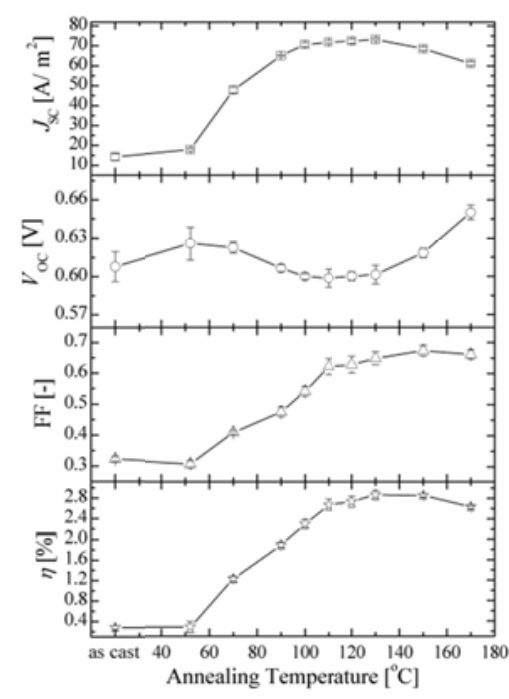

Figure 6. Device performance of the 50:50 wt.\% RR-P3HT:PCBM blends, under illumination from a halogen lamp, as a function of the annealing temperature. The annealing was done on a 
hot plate in $\mathrm{N}_{2}$ atmosphere for 4 min. The thickness of all devices ranges from 94 to $97 \mathrm{~nm}$. Reproduced from Adv. Funct. Mater. 2006, 16, 699-708.

Conversely, all remaining device parameters showed a very fast increase with annealing temperature between 50 and $110{ }^{\circ} \mathrm{C}$, followed by saturation. The onset of this enhancement strongly corresponds to the onset of the increase in hole mobility of RR-P3HT in the blend (compare Figure 3). Furthermore, the saturation of $J_{\mathrm{SC}}, \mathrm{FF}$ and $\eta$ upon annealing above 110 ${ }^{\circ} \mathrm{C}$ appears also to be related to the $\mathrm{P} 3 \mathrm{HT}$ hole mobility. Above this annealing temperature the enhancement of the hole mobility of P3HT in the blend is maximized, and consequently the difference in the electron and hole mobility is reduced typically by a factor of 20 , leading to a much more balanced charge carrier transport.

An important question to address is whether the unbalanced transport limits the performance of an as-cast device or a device annealed at lower temperature. It has been demonstrated that the photocurrent in polymer:fullerene blends can be limited by the build-up of space-charge, even under normal operation conditions equivalent to 1 sun illumination. This space-charge limited photocurrent occurs when the difference between electron and hole mobility is significant. ${ }^{[31]}$ The fingerprints of a SCL photocurrent are its square-root dependence on voltage and a three-quarter dependence on light intensity. ${ }^{[31]}$ The mobility measurements presented in Figure 3 show that there is more than a two orders of magnitude difference between the electron and hole mobility in as-cast devices and devices annealed up to $90{ }^{\circ} \mathrm{C}$. As expected, the photocurrents shown in Figure 1 indeed exhibit a square-root dependence on voltage for both the as-cast film and the device annealed at $70{ }^{\circ} \mathrm{C}$. To gain further insight in the operation of P3HT:PCBM devices and quantify the limiting parameters, the light intensity $\left(P_{\text {light }}\right)$ dependence of the photocurrent has been studied for the device annealed at $70{ }^{\circ} \mathrm{C}$. The $P_{\text {light }}$ was varied from $1000 \mathrm{~W} / \mathrm{m}^{2}$ (upper curve) down to $76 \mathrm{~W} / \mathrm{m}^{2}$ using a set of neutral density filters. Qualitatively, $J_{\mathrm{ph}}$ follows a power law dependence: 


$$
J_{\text {ph }} \propto P_{\text {light }}^{S},
$$

where the exponent $S$ ranges from 0.75 in the case of entirely space-charge limited photocurrent to 1.0 for the space-charge-free limit. ${ }^{[31]}$

a)

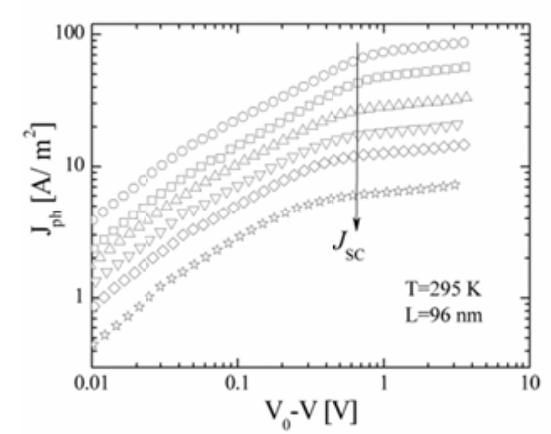

b)

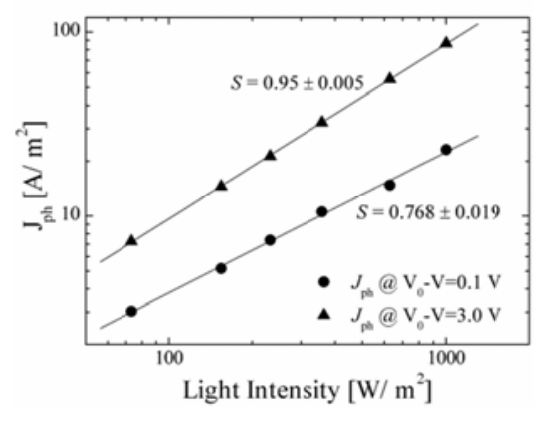

Figure 7. a) Light intensity dependence of the photocurrent $\left(J_{\mathrm{ph}}\right)$ versus effective applied voltage $\left(V_{0}-V\right)$ of the device thermally annealed at $70{ }^{\circ} \mathrm{C}$. The intensity was varied from 1000 (upper curve) down to $76 \mathrm{~W} / \mathrm{m}^{2}$, and the arrow indicates the position of the short-circuit current $\left(J_{\mathrm{SC}}\right)$ condition. b) Light intensity dependence of the photocurrent $\left(J_{\mathrm{ph}}\right)$ taken from Figure 6a at an effective voltage of $V_{0^{-}} V=0.1 \mathrm{~V}$ and $V_{0^{-}} V=3.0 \mathrm{~V}$ (symbols). The slope $(S)$ determined from the linear fit (solid lines) to the experimental data is denoted in the figure. Reproduced from Adv. Funct. Mater. 2006, 16, 699-708.

Figure 7a shows the $J_{\mathrm{ph}^{-}}\left(V_{0^{-}} V\right)$ characteristics of a RR-P3HT:PCBM device after thermal annealing at $70{ }^{\circ} \mathrm{C}$ as a function of the light intensity $P_{\text {light. }}$ For $V_{0^{-}} V<0.03 \mathrm{~V}$ the $J_{\text {ph }}$ showed a linear dependence on voltage at all light intensities, which is caused by the counteracting effect of drift and diffusion of charge carriers. ${ }^{[29-31]}$ Above $0.03 \mathrm{~V}$, however, a square-root dependence of the experimental $J_{\mathrm{ph}}$ on voltage was observed, as predicted for 
blends with a large difference in electron and hole mobilities. ${ }^{[31]}$ At even larger voltages the $J_{\text {ph }}$ showed a clear transition to the saturation regime, which was modeled by a field- and temperature dependence of the dissociation of bound electron-hole pairs according to $J_{\mathrm{ph}}=q G(\mathrm{E}, \mathrm{T}) L .^{[30]}$ These results are distinctly different when the devices were annealed at higher temperature, where the electron and hole transport became more balanced. In that case, the square-root dependence of $J_{\text {ph }}$ was no longer observed, as indicated in Figure 1 by the curve at $120{ }^{\circ} \mathrm{C}$. Moreover, it appears from Figure 6 a that $J_{\text {ph }}$ showed a weaker light intensity dependence in the square-root regime compared to the saturation regime. Figure $\mathbf{7 b}$ displays, on a double-logarithmic scale, the experimental $J_{\mathrm{ph}}$ taken from Figure $7 \mathrm{a}$ as a function of light intensity for two different voltages, that are $V_{0^{-}} V=0.1 \mathrm{~V}$ in the square-root regime and at $V_{0^{-}}$ $V=3 \mathrm{~V}$ in the saturation regime. The slope $S$ determined from the linear fit (solid lines) to the experimental data (as shown in Figure 6b) clearly proved that $J_{\mathrm{ph}}$ was limited by the build-up of space-charge in the square-root regime and became space-charge-free in the saturation regime. ${ }^{[31]}$ Because the short circuit current $J_{\mathrm{SC}}$ (indicated by the arrow in Figure 6a) at high light intensity lies in the square-root part of the $J_{\mathrm{ph}}-\left(V_{0}-V\right)$ characteristics, the device is entirely limited by the build-up of space-charge between the open- and short circuit point. In this case the FF of the device is reduced to less than 0.42 and the $J_{\mathrm{SC}}$ approaches the threequarters light intensity dependence $(\mathrm{S} \approx 0.75)$. By decreasing the light intensity, the transition voltage from a square-root dependence to the saturation limit shifted to lower values and the device was only partially limited by space-charge effects. ${ }^{[31]}$ As a consequence, the FF of the device increased and the $J_{\mathrm{SC}}$ approached a linear dependence on light intensity $(\mathrm{S} \approx 1)$.

From Figure $7 \mathrm{a}$ it is clear that the build-up of space charge dramatically reduced the device performance, since it caused a fundamental limitation of FF and $J_{\mathrm{SC}}$. This space charge is a direct result of the unbalanced transport of electrons and holes in the device. Due to the strongly increased hole mobility, leading to more balanced charge transport, this limitation was prevented under normal operation conditions in the device previously annealed above 
$110{ }^{\circ} \mathrm{C}$. With improved hole transport the devices recovered from the space-charge limitation and became space-charge-free, as a direct result of a more balanced transport. As shown in Figure 1 , the absence of the square-root voltage dependence in the device annealed at $110{ }^{\circ} \mathrm{C}$ led to a pronounced enhancement of both $\mathrm{FF}$ and $J_{\mathrm{SC}}$.

In subsequent work the photocurrent generation in RR-P3HT:PCBM solar cells was further quantified with the aid of numerical simulations. ${ }^{[29]}$ A process considered in these simulations is the dissociation probability $[P(\mathrm{E}, \mathrm{T})]$ of electron-hole pairs at the donoracceptor (D/A) interface, which is considered to be a field- $(\mathrm{E})$ and temperature $(\mathrm{T})$ dependence process. ${ }^{[30]}$ In the saturation regime the photocurrent is given by $J_{\mathrm{ph}}=q G(\mathrm{E}, \mathrm{T}) L$, in which $G(\mathrm{E}, \mathrm{T})=P(\mathrm{E}, \mathrm{T}) G_{\max }$. The dissociation probability $P$ is calculated using the OnsagerBraun model that takes into account the initial electron-hole separation distance $(a)$ and the decay rate of bound pairs $\left(k_{\mathrm{F}}\right)$. Once separated, the free electron and hole can again recombine non-geminately at the interface to (re)form a bound pair with a rate constant $\left(k_{\mathrm{R}}\right)$, which may dissociate again during its lifetime. To fit the experimental data, the $G(\mathrm{E}, \mathrm{T})$ had been taken into account in a numerical model which solves the steady-state continuity equations for electrons and holes including diffusion, recombination and space-charge effects via the Poisson equation. ${ }^{[29]}$ To calculate the photocurrent for the RR-P3HT:PCBM devices, the input parameters required in the model are: the charge carrier mobility of electrons and holes, the spatially averaged dielectric constant $<_{\mathcal{E}_{\mathrm{P}}}>$ of P3HT and PCBM, the initial (electron-hole) separation distance $a$, the bound pair lifetime $\left(k_{\mathrm{F}}^{-1}\right)$, the maximum generation rate $G_{\max }$, and the semiconductor band gap $\left(E_{\mathrm{g}}\right)$. Since $\varepsilon_{\mathrm{r}}$ for P3HT and PCBM are known, the $E_{\mathrm{g}}$ is estimated from the $\mathrm{HOMO}(\mathrm{P} 3 \mathrm{HT})-\mathrm{LUMO}(\mathrm{PCBM})$ difference, $G_{\max }$ is determined from the saturation of the photocurrent, and the charge carrier mobilities are known as well (compare Figure 3), the only adjustable parameters of the model that remain are $a$ and $k_{\mathrm{F}}{ }^{-1}$. Figure 8 shows the temperature dependence of the $J_{\mathrm{ph}}$ versus $V_{0^{-}} V$ of a RR-P3HT:PCBM device after thermal annealing at $120{ }^{\circ} \mathrm{C}$. It appears that the experimental $J_{\mathrm{ph}}$ exhibits an extremely weak 
field- and temperature dependence in the saturation regime for $V_{0^{-}} V>0.3 \mathrm{~V}$, where $J_{\mathrm{ph}}=q P(\mathrm{E}, \mathrm{T}) G_{\max } L$. For effective voltages $V_{0}-V>3 \mathrm{~V}$, the experimental $J_{\mathrm{ph}}$ clearly approached full saturation meaning that all electron-hole pairs are fully dissociated $(P \rightarrow 1)$ and $J_{\text {sat }}=q G_{\text {max }} L$. By comparing the $J_{\text {ph }}$ with the experimentally observed $J_{\text {sat, }}$, the dissociation probability at any effective voltage can be read directly from the experimental data. For example, under short-circuit condition $(V=0 \mathrm{~V})$, the dissociation probability of electron-hole pairs $\left(P_{\mathrm{SC}}\right)$ is close to 0.9 (at room temperature), that is considerably larger than previously reported for MDMO-PPV:PCBM devices $\left(P_{\mathrm{SC}}=0.6\right) .{ }^{[30]}$ This high internal quantum efficiency is in perfect agreement with the results reported by Burkhard et al. ${ }^{[48]}$ and the $20 \%$ loss by geminate recombination of CT-states demonstrated by TA experiments of Howard et al. ${ }^{[51]}$

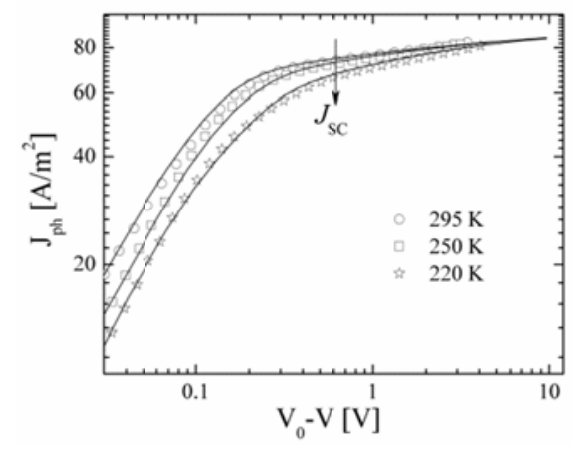

Figure 8. Temperature dependence of the photocurrent (symbols) versus effective applied voltage $\left(V_{0}-V\right)$ for a 50:50 wt.\% RR-P3HT:PCBM device with a thickness of $120 \mathrm{~nm}$, annealed at $120{ }^{\circ} \mathrm{C}$ for $4 \mathrm{~min}$. The solid lines represent numerical calculations using the model from Ref. [29].

The solid lines in Figure 8 represent the numerical calculation of $J_{\mathrm{ph}}$ including the field- and temperature dependent generation rate $G(\mathrm{E}, \mathrm{T})$. From the best fit to the experimental data an electron-hole separation distance of $a=1.8 \mathrm{~nm}$ and room temperature bound pair decay lifetime of $k_{\mathrm{F}}^{-1} \approx 50 \mu$ s were obtained. Compared to the MDMO-PPV:PCBM system it was deduced that both $a$ and $k_{\mathrm{F}}{ }^{-1}$ are larger, ${ }^{[28,30]}$ thereby leading to an enhanced dissociation 
probability. Because of this efficient dissociation, the field-dependence of $J_{\text {ph }}$ is extremely weak, which in turn means in terms of photocurrent-voltage characteristics, that it is possible to obtain a FF as high as 0.7 , as, in fact, is observed experimentally (Figure 6). The very weak dependence of the photocurrent on temperature and electric field is also in agreement with the observation in TA experiments that reducing the temperature of the blends to $80 \mathrm{~K}$ did not have an effect on the splitting ratio between free charge carriers and charge-transfer excitons for both RR-P3HT:PCBM and RRa-P3HT:PCBM. ${ }^{[54]}$ This implies that the free energy difference between the polymer exciton and the free charges is sufficient to drive charge separation even at cryogenic temperatures. In addition, recent time-delayed collection field (TDCF) experiments addressing the field dependence of charge separation in P3HT:PCBM by the Neher group convincingly demonstrated field-independent charge separation in as-cast and annealed blends. ${ }^{[55]}$

It should be noted that the inverse recombination rate used in the Onsager-Braun model required to fit the bias dependence of experimentally measured J-V-curves are often on the order of hundreds of nanoseconds, sometimes even microseconds, and thus are incompatible with the geminate recombination rates determined by spectroscopy. Hence, the OnsagerBraun model should rather be considered a parametrization of the experimental data than reflecting the underlying physics of the charge separation process. However, the physics behind the charge separation process is still controversially discussed and out of the scope of this review.

\subsection{Charge Carrier Recombination in P3HT:PCBM}

Geminate recombination of charge transfer states is intrinsically included in the charge generation rate, while non-geminate recombination of free charge carriers occurs after charges have been fully separated. The prerequisite of non-geminate recombination of spatiallyseparated charge carriers is their encounter in the photoactive medium. In pristine organic semiconductors bimolecular recombination is of Langevin-type, that is, the rate-limiting step 
of the recombination is the diffusion of electrons and holes toward each other and the capture in their mutual Coulomb field. The Langevin bimolecular recombination coefficient is given by

$$
\gamma_{L}=\frac{q}{\varepsilon}\left(\mu_{n}+\mu_{p}\right)
$$

with $\mu_{n}$ and $\mu_{p}$ the electron and hole mobilities, respectively. The bimolecular Langevin recombination rate then yields $R_{L}=\gamma_{L}\left(n p-n_{i}^{2}\right)$, where $n(p)$ is the free electron (hole) density and $n_{i}$ is the intrinsic carrier density. Langevin-type recombination is characteristic of materials in which the mean free path of the charge carriers is smaller than a critical distance $r_{c}=q^{2} / 4 \pi \varepsilon_{0} \varepsilon_{r} k T$, with $q$ the elementary charge, $\varepsilon_{0}$ and $\varepsilon_{r}$ the vacuum permittivity and dielectric constant of the medium, respectively, and $T$ the absolute temperature, at which the Coulomb binding energy between an electron and hole equals $k T$. As stated above charge transport in organic semiconductors is of a hopping nature, with a typical hopping distance of $1-2 \mathrm{~nm}$, whereas $r_{c}$ amounts to $18.5 \mathrm{~nm}$ at $\mathrm{T}=300 \mathrm{~K}$ for $\varepsilon_{r}=3$. Therefore, the manifestation of Langevin recombination in organic semiconductors is expected. Indeed, from a first glance, as observed in MDMO-PPV:PCBM solar cells, the recombination is equivalent to a Langevintype non-geminate recombination process. ${ }^{[56]}$

However, in a bulk heterojunction solar cell charges can only recombine at the interface between donor and acceptor. Therefore, the recombination coefficient $\gamma$ is not necessarily equal to the calculated Langevin coefficient $\gamma_{L}$ and in fact often is reduced. Furthermore, in a solar cell an equal amount of electrons $(n)$ and holes $(p)$ is created, thus $p=n$. Hence, carrier recombination follows a modified (reduced) Langevin recombination rate model according to the following rate equation: 
$\frac{d n}{d t}=-\gamma(n) n^{2}=-\xi \frac{e\left(\mu_{n}+\mu_{p}\right)}{\varepsilon_{0} \varepsilon_{r}} n^{\lambda+1}$,

in which $\gamma$ is the recombination coefficient, $n$ is the charge carrier concentration, $\xi$ is a reduction prefactor, $e$ is the elementary charge, and $\lambda+1$ is the total recombination order, while the other parameters have their usual meaning is described above. In fact, the recombination rate $\gamma$ differs from the traditional Langevin rate in two ways. Firstly, the rate constant is not independent of the charge carrier density. The total recombination order determined for annealed RR-P3HT:PCBM blends is increased by 0.45 , that is to $\lambda+1=$ $2.45,{ }^{[51]}$ compared to a simple two-body Langevin recombination mechanism. The higher reaction order can have two origins. On the one hand Juska et al. have shown that when charge transport is limited to two dimensions, the order of the recombination process increases to $2.5 .^{[56]}$ The tendency of regioregular P3HT to align into well-ordered lamellar structures could indeed explain a preferential charge transport in two dimensions. However, other studies have shown that the non-geminate recombination order is heavily influenced by the processing conditions ${ }^{[57]}$ and also varies between different donor polymers used. ${ }^{[58]}$ In addition, the total recombination order increases with decreasing temperature as shown by temperature dependent TA measurements. ${ }^{[54]}$ This cannot be explained by a limitation of the dimensionality of charge transport. On the other hand, an increased recombination order can be a consequence of a charge carrier density dependent charge carrier mobility. In fact, Shuttle et al. recently investigated the charge carrier density dependence of both the nongeminate recombination rate and the charge carrier mobility in RR-P3HT:PCBM solar cells and observed virtually identical dependencies for both parameters, indicating that indeed the dependence of the charge carrier mobility on the carrier concentration is responsible for the density dependence of the recombination rate. ${ }^{[59]}$ In fact, at lower temperatures a more pronounced dependence of the mobility on the charge carrier concentration is expected ${ }^{[19]}$ in 
turn leading to a higher recombination order in agreement with temperature dependent TA measurements. ${ }^{[54]}$

The second deviation from the classical Langevin recombination is the dimensionless reduction prefactor $\xi$. Using a charge carrier mobility of $5 \times 10^{-5} \mathrm{~cm}^{2} \mathrm{~V}^{-1} \mathrm{~s}^{-1}$ for both electrons and holes as determined by TOF experiments for RR-P3HT:PCBM and a relative permittivity of 3.5 yields a Langevin recombination rate of $\gamma=5.2 \times 10^{-11} \mathrm{~cm}^{3} \mathrm{~s}^{-1}$. Experimentally, rate constants corresponding to a second order recombination process of $3 \times 10^{-13} \mathrm{~cm}^{3} \mathrm{~s}^{-1}$ for annealed RR-P3HT:PCBM and $1.7 \times 10^{-12} \mathrm{~cm}^{3} \mathrm{~s}^{-1}$ for as-cast RR-P3HT:PCBM at a charge carrier concentration of $10^{16} \mathrm{~cm}^{-3}$ were found, ${ }^{[51]}$ that is a factor $\xi=5.8 \times 10^{-3}$ and $3.4 \times 10^{-2}$ lower than the calculated value of $\gamma$, respectively. These values obtained by TA experiments are well in line with recent experimental results presented by several groups. ${ }^{[60-62]}$ The reduction of the recombination rate constant is an inherent property of the bulk heterojunction structure of the photoactive layer. In fact, Langevin theory was developed for recombination of charge carriers in a homogeneous and isotropic medium. In a bulk heterojunction however, the holes and electrons are confined to the donor and the acceptor phase, and thus can only recombine, if they encounter at the interface. This in turn reduces the probability for recombination effectively leading to a reduction of the non-geminate rate constant. The reduction factor $\xi$ used to modify the Langevin equation is therefore closely related to the morphology of the blend and the effective interfacial area. The observation that annealing causes a reduction of the recombination rate by one order of magnitude suggests that it leads to a higher degree of demixing and thus to a reduced interfacial area. The effect of external stimuli on the recombination rate mainly follows the charge transport dependencies. Fielddependent TOF measurements demonstrated a weak field dependence of the charge carrier mobility hence, the recombination rate itself can be considered independent of the applied electric field. ${ }^{[41]}$ However, this does not mean that the non-geminate recombination rate does not vary with the electric field in a solar cell. In fact, the steady-state charge carrier density in 
a solar cell strongly depends on the applied bias and in turn the recombination rate changes with the charge carrier concentration in the device. The temperature dependence of the charge carrier mobility for instance determined by TOF experiments can explain the reduction of the recombination rate constant by two orders of magnitude when going from room temperature to $80 \mathrm{~K}$.

\subsection{Charge Carrier Extraction in P3HT:PCBM Blends}

Let us start with reviewing some basic considerations of the carrier extraction mechanism in organic photovoltaic devices. The charge carrier extraction from the photoactive layer is governed by the charge transport properties of the blend, that is mostly by the charge carrier mobility. A directed movement of charge carriers is a consequence of drift of charges in the internal electric field or Fickian diffusion due to carrier concentration gradients in the photoactive layer. Generally, both effects have to be considered when modeling the dependence of the photocurrent density on the device bias and thus a complete description of the bias dependence of the photocurrent requires the use of numerical drift-diffusion models. ${ }^{[29]}$ However, neglecting the diffusional contribution to charge transport allows an analytic description of the field dependence of the photocurrent at the cost of a precise description at low biases, that is close to the open circuit voltage, where diffusion is dominant over drift. ${ }^{[63]}$ In the following this simplification was used to illustrate some basic dependencies. Apart from the limiting case of low electric fields, the analytical model can yield insight into the processes that govern the field-dependence of the photocurrent and it is much more intuitive than a drift-diffusion model. Assuming that charge extraction is exclusively driven by the drift of carriers in the electric field and neglecting any fielddependence of the charge carrier mobility, which is safe for P3HT:PCBM in the low field regime that solar cells typically operate in, the charge carrier extraction rate can be expressed as follows: 
$k_{\text {extr }}(F, T)=\frac{2}{d} \mu(T) F$

Here, a factor of 2 simply accounts for the fact that, charge carriers have to travel on average a distance equal to half of the thickness $d$ of the photoactive layer, before being extracted at an electrode, if a homogeneous charge generation profile throughout the photoactive layer is assumed. The value expected for $k_{\text {extr }}$ in annealed RR-P3HT:PCBM can be estimated to $k_{\text {extr }}=$ $6 \times 10^{5} \mathrm{~s}^{-1}$ from the charge carrier mobility $\mu=5 \times 10^{-5} \mathrm{~cm}^{2} \mathrm{~V}^{-1} \mathrm{~s}^{-1}$ as obtained by time-of-flight measurements and the internal electric field of an organic solar cell close to short circuit conditions as given by $F=U / d=0.6 \mathrm{~V} / 100 \mathrm{~nm}=6 \times 10^{4} \mathrm{~V} / \mathrm{cm}$. In fact, the extraction rate is the only field-dependent parameter in the description of the photocurrent of annealed RRP3HT:PCBM solar cells, if one assumes a field-independent charge carrier mobility and a field-independent free charge carrier generation yield as demonstrated by time-of-flight and time-delayed collection field experiments, respectively. ${ }^{[41,55]}$ Using the expressions for the extraction rate constant $k_{\text {extr }}$ and $\gamma=\xi\left(\mu_{n}+\mu_{p}\right) e /\left(\varepsilon_{0} \varepsilon_{r}\right) \approx 2 \xi \mu e /\left(\varepsilon_{0} \varepsilon_{r}\right)$ yields the following field dependence of the photocurrent $J_{\text {photo }}$ :

$J_{\text {photo }}(F)=-\frac{\varepsilon_{0} \varepsilon_{r}}{d} \frac{\mu}{\xi} F^{2}\left[\sqrt{1+\frac{2 e d^{2}}{\varepsilon_{0} \varepsilon_{r}} \frac{\xi}{\mu} \frac{G}{F^{2}}}-1\right]$

Clearly, increasing the charge carrier mobility has the same beneficial effect on the photocurrent as decreasing the prefactor $\xi$ of the non-geminate recombination rate. Hence, from the point of device performance increasing the charge carrier mobility, while decreasing the interfacial area available for charge recombination, is beneficial. However, decreasing the interfacial area may not only decrease the non-geminate recombination rate, but also the exciton quenching efficiency. This implies an optimum morphology exists, that provides a 
balance between efficient exciton quenching and charge separation on the one hand and reduced non-geminate recombination on the other hand.

Figure 9 shows the voltage dependence of the photocurrent of a P3HT:PCBM solar cell assuming a charge generation rate of $\mathrm{G}=6.26 \times 10^{21} \mathrm{~cm}^{-3} \mathrm{~s}^{-1}$ (see above) plotted for different ratios of the prefactor $\xi$ and the charge carrier mobility $\mu$. The electric field $F=\left(V_{a p p l}-V_{B I}\right) / d$ used in this calculation was calculated from the effective voltage, that is the difference between the built-in voltage $V_{B I}(0.7 \mathrm{~V})^{[54]}$ and the externally applied voltage $V_{\text {ext }}$, and the active layer thickness $d$ :

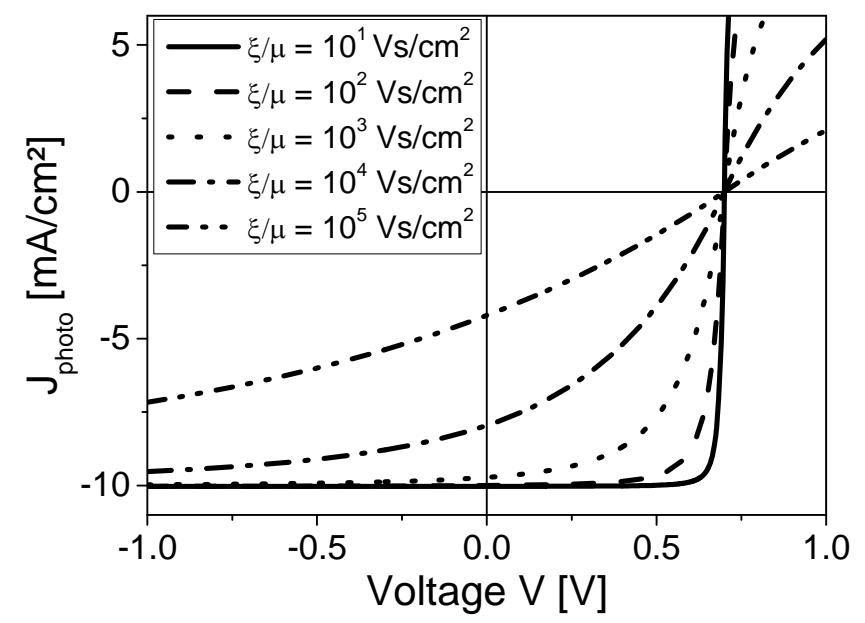

Figure 9. Voltage dependence of the photocurrent of a solar cell for various ratios of the reduction prefactor $\xi \mu$ and the charge carrier mobility $\mu$ assuming a charge generation rate of $\mathrm{G}=6.26 \times 10^{21} \mathrm{~cm}^{-3} \mathrm{~s}^{-1}$. The electric field is calculated from the applied and built-in voltage as $F=\left(V_{a p p l}-V_{B I}\right) / d$ with $V_{B I}=0.7 \mathrm{~V}$.

The calculated data indicates that at a high reverse bias such as $-1 \mathrm{~V}$ essentially all charge carriers are extracted except for the highest $\xi / \mu$ ratio shown here. However, when lowering the electric field by going from a high reverse bias towards the built-in voltage, not all charge carriers are extracted anymore and non-geminate recombination sets in. Thus, the fraction of charge carriers lost due to non-geminate recombination at any given voltage depends on the $\xi / \mu$ ratio.

In P3HT:PCBM this ratio depends on the morphology on different length scales. On the one hand the regioregularity determines the packing of the polymer chains within the P3HT 
domains. ${ }^{[41]}$ This results in a drastic variation of the charge carrier mobility even for small changes in the regioregularity. In fact, the hole mobilities of polymers with regioregularities of $>98 \%$ and $94 \%$ differ by one order of magnitude with the higher mobility for the higher regioregularity. ${ }^{[41]}$ In samples of as-cast RR-P3HT:PCBM the order of P3HT chains is much less and consequently the hole mobility is significantly lower than in annealed samples (vide supra). ${ }^{[22]}$ The packing of the P3HT chains influences the charge transport and thus the $\xi / \mu$ ratio. Besides, the degree of demixing plays an important role. The miscibility of PCBM molecules with amorphous P3HT tends to be higher than with well-ordered more semicrystalline P3HT domains, likely due to size exclusion in the tightly-packed lamellar structure of well-ordered P3HT chains suppressing PCBM intercalation. ${ }^{[51,64,65]}$ In general this implies that blends of well-ordered RR-P3HT and PCBM exhibit a higher degree of demixing and only limited intermixing in the more amorphous regions of the polymer film and close to the interface. The degree of demixing and the extent of intermixing are thus related to the regioregularity of P3HT. Annealed $>98 \%$ RR-P3HT:PCBM shows a high degree of demixing with very limited intermixing, while RRa-P3HT:PCBM lacks demixing almost entirely and rather consists of finely-dispersed PCBM in an amorphous RRa-P3HT matrix. Consequently, the interfacial areas differ extensively between the two system as does the reduction factor $\xi$. In annealed RR-P3HT:PCBM the ratio of the reduction factor to the charge carrier mobility is $\sim 10^{2} \mathrm{Vs} / \mathrm{cm}^{2}$, which corresponds to the dashed line in Figure 9. At this ratio charge extraction from the photoactive layer at short circuit conditions is almost quantitative. In fact, timedelayed double-pump (TDDP) experiments reported by Mauer et al. demonstrated that at short circuit conditions only $2 \%$ of the free charges undergo non-geminate recombination prior to extraction (see Figure 10). ${ }^{[66]}$ The fraction of non-geminate recombination increases towards open circuit conditions and in turn determines the fill factor of these devices. 


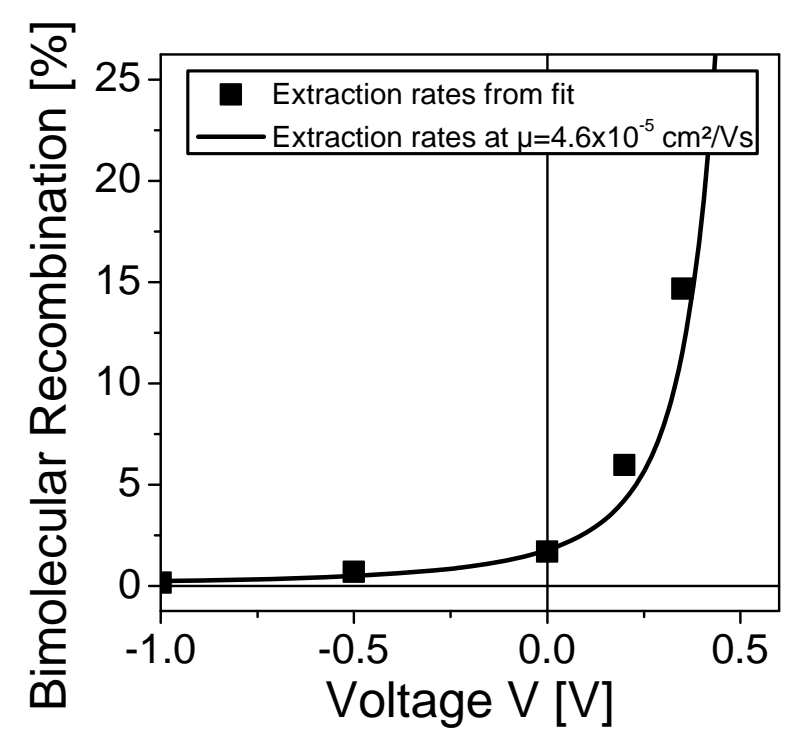

Figure 10. Fraction of nongeminate recombination in a RR-P3HT:PCBM device as a function of the external bias recalculated to steady-state conditions from time-delayed double pump experiments. The solid line is a fit using an effective charge carrier mobility of $4.6 \times 10^{-5} \mathrm{~cm}^{2}$ $\mathrm{V}^{-1} \mathrm{~s}^{-1}$ to determine the extraction rates. Reprinted from Mauer et al., J. Phys. Chem. Lett. 2011, 2, 1736 .

When cooling annealed RR-P3HT:PCBM to $80 \mathrm{~K}$ thereby reducing the charge carrier mobility by approximately 2 orders of magnitude, the $\xi / \mu$ ratio is increased to $10^{4} \mathrm{Vs} / \mathrm{cm}^{2}$ (dash-dotted line in Figure 9). This is in line with the temperature dependent reduction of the device performance of annealed RR-P3HT:PCBM solar cells observed at low temperatures. ${ }^{[54]}$ In as-cast RR-P3HT:PCBM this ratio is even higher. Despite the hole mobility being much lower than in the annealed case ${ }^{[22]}$ the effective recombination rate is almost an order of magnitude higher, which demonstrates that the reduction factor must also be much bigger than in annealed RR-P3HT:PCBM. In fact, using the values of the charge carrier mobility determined by Mihailetchi et al. ${ }^{[22]}$ for as-cast RR-P3HT:PCBM the ratio $\xi / \mu$ is estimated to be $\sim 10^{5} \mathrm{Vs} / \mathrm{cm}^{2}$ (corresponding to the dash double-dotted line in figure 9), in part explaining the poor performance of as-cast RR-P3HT:PCBM devices. 


\section{Theoretical studies of hole transport in P3HT and P3HT:PCBM mixtures}

In this section we review theoretical interpretations and simulation results related to charge transport in both pristine $\mathrm{P} 3 \mathrm{HT}$ and $\mathrm{P} 3 \mathrm{HT}: \mathrm{PCBM}$ mixtures. It has already been mentioned that $\mathrm{P} 3 \mathrm{HT}$ exhibits reasonable charge mobilities ${ }^{[67]}$ which depend on polymer regioregularity, molecular weight, and processing conditions. Hole mobilities of $10^{-5} \mathrm{~cm}^{2} / \mathrm{Vs}\left(10^{-4} \mathrm{~cm}^{2} / \mathrm{Vs}\right)$ were measured for $94 \%(98 \%)$ regioregular P3HT using the time-of-flight (TOF) technique ${ }^{[41]}$ Dispersive transients of regiorandom P3HT indicated that the polymer is conductive, yet its mobility could not be extracted from TOF measurements due to sizeable disorder. The mobility-temperature dependence, analyzed using the Gaussian disorder model (GDM), suggested an energetic disorder on the order of $50-60 \mathrm{meV}$. Both hole and electron TOF mobilities were reported to be independent of the molecular weight up to $20 \mathrm{kDa}$, which then decreased by an order of magnitude as molecular weight was further increased to $120 \mathrm{kDa}^{[68]}$ The reported zero-field mobilities for shorter chains were of the order of $10^{-4} \mathrm{~cm}^{2} /$ Vs. A GDM-fitted energetic disorder of $71(54) \mathrm{meV}$ was extracted for short (long) chains. Meanwhile, field-effect mobilities ranging around $10^{-5} \mathrm{~cm}^{2} / V s$ were reported for OFET devices. ${ }^{[69]}$ By using regioregular P3HT, OFET mobility could be increased by three orders of magnitude. ${ }^{[70]}$ Moreover, OFET mobilities of $\sim 0.1 \mathrm{~cm}^{2} /$ Vs were measured after spin-casting from higher boiling point solvents. ${ }^{[71]}$ The field-effect mobility was found to increase with molecular weight in spite of reduced semi-crystallinity. This was attributed to either better interconnectivity of the polymer network ${ }^{[72]}$ or smaller intrachain ring torsions present in high molecular weight molecules. ${ }^{[7]}$ Transistor hole mobilities have even been reported for P3HT nanofibers. ${ }^{[74-76]}$ Along the fiber this mobility was as high as $0.06 \mathrm{~cm}^{2} / \mathrm{Vs}$, and energetic disorder of $108 \mathrm{meV}$ was extracted from temperature-dependent measurements. Stipulated by the multitude of experimental investigations, a number of theoretical studies have been conducted to rationalize the wide spectrum of mobilities obtained for a single compound and to link the transport characteristics to the self-assembly properties, 
morphology, and electronic structure of P3HT. The controversy started already when examining conformations of an isolated chain, where the thiophene dimer was reported to adopt a twisted backbone conformation, ${ }^{[77]}$ while increasing the oligomer length results in a planarized backbone. The situation with oligomer assemblies turned out to be even more complex. To this end, we do not know the order of semi-crystalline polymorphs on the energy axis and cannot quantify the density of defects in a semi-crystalline morphology or relative volume fractions of crystalline and amorphous phases. We do not really understand whether large energetic disorder or small electronic couplings is the main transport-limiting factor and cannot rationalize the dispersive, in spite of a moderate energetic disorder, nature of charge transport. The goal of this chapter is to outline approaches and answers to some of these questions and to provide an outlook for the rest of them.

We will first discuss the morphology of a pristine P3HT and a P3HT:PCBM mixture, since it is known to have a large impact on charge transport as well as optical properties of these materials. As many conjugated polymers, P3HT is a polymorph, that is, forms different crystal structures depending on processing conditions. The most frequently observed are socalled forms I and II, ${ }^{[78]}$ which differ by the side chain conformation and interdigitation, tilt of conjugated backbones with respect to the stacking direction, and the shift of successive (along the $\pi$-stacking direction) polymer chains. ${ }^{[79]}$ Form I, which is observed after annealing, is the structure encountered in most studies dealing with OFETs and OPVs. It has a monoclinic unit-cell (for bulk P3HT samples XRD provides $a=1.6 \mathrm{~nm}, b=0.78 \mathrm{~nm}, c=0.78 \mathrm{~nm}$ ). ${ }^{[80]}$ Polymorph II has been found to have a significantly smaller unit cell dimension along the $a$ axis and has thus been assumed to have interdigitated alkyl groups,${ }^{[78]}$ which distinguishes it from form I. Upon heating, form II irreversibly transforms into form I. This phase transition is accompanied by a change in unit-cell dimensions, with the interlayer spacing increasing and the intrastack distance decreasing. A similar first-order phase transition has been described recently by a combined infrared-spectroscopy and wide-angle X-ray diffraction study for a 
non-interdigitated metastable polymorph that transforms into the stable form I, hence establishing a third polymorph I' ${ }^{[81,82]}$

The goal of theoretical and computational techniques is to self-assemble the polymer in silico, that is, to predict its polymorphs as well as the degree of disorder in the kinetically trapped molecular arrangements. The computational toolbox developed for this purpose is very versatile: On the highest level of resolution, it includes accurate quantum chemical calculations capable of predicting properties of isolated oligomers and dimers, normally without side chains. Less computationally demanding density functional methods can deal with much longer oligomers (10-20 repeat units), including side chains, and are often used to compare ground state energies of experimentally proposed arrangements of atoms in a unit cell. To assess crystalline packing modes at ambient conditions and during annealing, as well as to study amorphous melts and longer chain lengths, classical force-fields have been parameterized. To reach even longer length- and timescales (micrometers, microseconds), coarse-grained models have been developed to study amorphous melts and liquid-crystalline phases of P3HT. In spite of such versatility, we are still unable to systematically study polymer self-assembly. The main obstacles are insufficient accuracy of methods at a specific level of resolution, long simulation times required to study self-assembly, and uncontrolled error propagation from one level to another, e.g., when parameterizing force-fields based on quantum chemical calculations, or developing coarse-grained models using force-fieldgenerated reference data. Below we provide a summary of simulation results, starting with single-molecule properties and then expanding to molecular arrangements of P3HT in crystals, melts, and finally binary mixtures with PCBM.

To this end, ab initio methods have been extensively used to analyze conformations of the conjugated backbone and side-chain orientations with respect to the plane of conjugation. ${ }^{\text {[77] }}$ Here, the extended $\pi$-conjugated system flattens the backbone, while non-bonded interactions between consecutive repeat units (that is, steric repulsions between hydrogens, Coulomb and 
van-der-Waals interactions) often tend to distort its planarity. For P3HT both planar ${ }^{[83]}$ and non-planar stable geometries have been reported, depending on the side-chain orientation. ${ }^{[84]}$ At the B3LYP/6-31+G** level of theory the non-planar backbone has an energy of $0.03 \mathrm{eV}$ lower (per monomer) than the planar backbone (evaluated in a 10 -mer). ${ }^{[85]}$ This indicates that chain conformations in the bulk are predominantly determined by interchain van-der-Waals and Coulomb interactions, a conclusion also drawn from calculations of molecular dimers. ${ }^{[86]}$ Since typical energy differences between planar and non-planar conformations are in the order of tens of meV, which is the accuracy threshold of density functional methods, one is forced to use more accurate (and computationally demanding) quantum-chemical methods. However, the ground-state twist angle between repeat units has been found to depend on the oligomer length, saturating at ca. 10 repeat units. Furthermore, torsional potentials are correlated up to the second-nearest-neighbor rings, ${ }^{[87]}$ thus making geometry predictions a formidable task even for isolated oligomers. ${ }^{[88]}$

Both density functional and force-field calculations have been used to study crystalline P3HT mesophases. Density functional calculations have been primarily used to establish whether experimentally reported crystal structures correspond to well-defined energy minima. ${ }^{[89-92]}$ Using a van-der-Waals corrected GGA functional, Dag and Wang concluded that the crystal with shifted backbones, that is with one of the thiophene layers shifted along the chain direction by the thiophene-thiophene distance, and side chains rotated around the torsion angle is the most stable among three studied structures. ${ }^{[93]}$ Xie et al. have reached the conclusion that a structural motif without this registry shift and without rotation of the side chains, but instead with a small backbone tilt has the lowest potential energy. ${ }^{[92]}$ Similar to the situation with a single isolated chain, typical energy differences between different packing motives are in the order of ten meV per unit cell and hence theoretical methods are at their accuracy limits, making it difficult to rank different molecular arrangements. Also, unit-cell 
optimizations are performed at zero Kelvin, hence entropic effects, notably the chain excluded volume, are ignored.

To study larger systems and longer timescales, various flavors of P3HT force fields were developed. In the majority of cases, parameters of an existing force field were refined in order to reproduce the torsional potential between thiophene units and electrostatic potential around an isolated oligomer. ${ }^{[94,95]}$ The parameterization has been subsequently refined to account for the change in the backbone potential with the oligomer length. ${ }^{[85,96,83,95,97]}$. To this end, atomistic simulations have been used to analyze proposed packing arrangements of three P3HT polymorphs, phases I, I', and II, and to scrutinize the effect of regioregularity on paracrystalline, dynamic, and static nematic order parameters. ${ }^{[95]}$ Molecular dynamics simulations predicted that the most stable P3HT polymorph has planar thiophene backbones shifted by one thiophene ring with respect to each other. Hexyl side chains are twisted away from the backbone in a trans fashion, resulting in a non-interdigitated packing structure, though stable structures with interdigitated side chains were also reported. ${ }^{[98]}$ Force-fieldbased estimates of the mass density $\left(1.05 \mathrm{~g} / \mathrm{cm}^{3}\right)$, melting temperature $(490 \mathrm{~K})$, and surface tension $(32 \mathrm{mN} / \mathrm{m})$ all agree with the reported experimental values ${ }^{[96]}$. One should note that even on this, classical, level of description, only highly crystalline morphologies and hightemperature amorphous melts can be studied. Still, some indications of local-chain ordering upon cooling of amorphous high-temperature melts have been observed. ${ }^{[99]}$

Early simulations of amorphous systems considered thiophene oligomers as a model for P3HT: Alignment of polymer chains and thiophene units within chains have for instance been studied in a Monte-Carlo approach. ${ }^{[100]}$ The authors were able to reproduce density of the amorphous mesophase (an estimate of $1.06 \mathrm{~g} / \mathrm{cm}^{3}$ was given) and concluded that chains tend to align parallel to each other, while thiophene rings of neighboring chains tend to adopt parallel or antiparallel $\pi$-stacked arrangements. Systems with much shorter chains had significantly denser packing (predicted density $1.4 \mathrm{~g} / \mathrm{cm}^{3}$ ) and stronger alignment, a result 
obtained using molecular-dynamics simulations. ${ }^{[101]}$ Amorphous melts of oligomers of P3HT were also simulated in order to calculate the glass transition temperature $(300 \mathrm{~K})$, hence validate the atomistic force-field ${ }^{[96]}$ and to develop coarse-grained models of P3HT in a liquid state approaching $500-600 \mathrm{~K} .^{[102,103]}$ Simulations of free-standing films of P3HT melts have been used to estimate the room-temperature value of surface tension $(21-36 \mathrm{mN} /$ m) ${ }^{[104]}$ Amorphous melts of P3HT are, however, of only moderate interest, since conductive abilities of this polymer are related to its high degree of lamellar ordering.

In order to understand molecular ordering and electronic processes in bulk heterojunction devices, blends of P3HT oligomers with fullerene and PCBM have been simulated at different levels of resolution. Atomistic simulations showed that bulk oligothiophenes (five chains of 20 repeat units each) tend to cluster better than oligothiophene/fullerene systems. ${ }^{[105]}$ Prototypical model interfaces have been used to evaluate energetic profiles for electrons and holes. ${ }^{[106]}$ Coarse-grained simulations could observe the onset of phase separation, ${ }^{[103]}$ distributions of domain sizes and interface-to-volume ratios ${ }^{[107]}$ in P3HT:PCBM mixtures. Nevertheless, the field of coarse-grained modeling of conjugated polymers is still in its infancy. Complications reside in the large persistence length and anisotropic non-bonded interactions that promote $\pi-\pi$ stacking. In addition, mesoscopic ordering of conjugated polymers is equally important for the functionality of organic semiconducting devices. In a bulk heterojunction solar cell, for example, domain sizes of the donor and acceptor mesophases have considerable impact on cell efficiency. To predict and analyze such effects, modeling strategies that target the morphology on length scales reaching several hundreds of nanometers, the typical thickness of the active layer in a polymer-based solar cell, are required. With the currently available computational power, such system sizes can only be addressed on a coarse-grained level. Structure-based coarse-grained models have been developed for melts, ${ }^{[102,103]}$ high-temperature (liquid) mixtures of P3HT:PCBM, ${ }^{[103,108,107]}$ and solutions of P3HT. ${ }^{[109]}$ These models allow for self-assembling polymer backbones into 
lamellar arrangements. ${ }^{[108,109]}$ They have also been used to study the morphology of phaseseparated P3HT:PCBM blends, where explicit incorporation of side chains helped to understand penetration of acceptor molecules into side chains as a function of their grafting density along the backbone. No intercalation was found for P.3HT, contrary to poly(bithiophene-alt-thienothiophene) (PBTTT), which has a lower grafting density of side chains. ${ }^{[108]}$ As a further advantage of coarse-graining, solvent-mediated interactions can be incorporated into coarse-grained interactions, leading to a dramatic decrease of the number of degrees of freedom. For example, a simulation of P3HT aggregation in solutions with anisole could reproduce the experimentally observed aggregation of P3HT as a function of temperature with the backfolded hairpins of stacked P3HT molecules. ${ }^{[110]}$ The spacing between P3HT lamellae was found to be $1.7 \mathrm{~nm}$, comparable to experimental observations. ${ }^{[79]}$

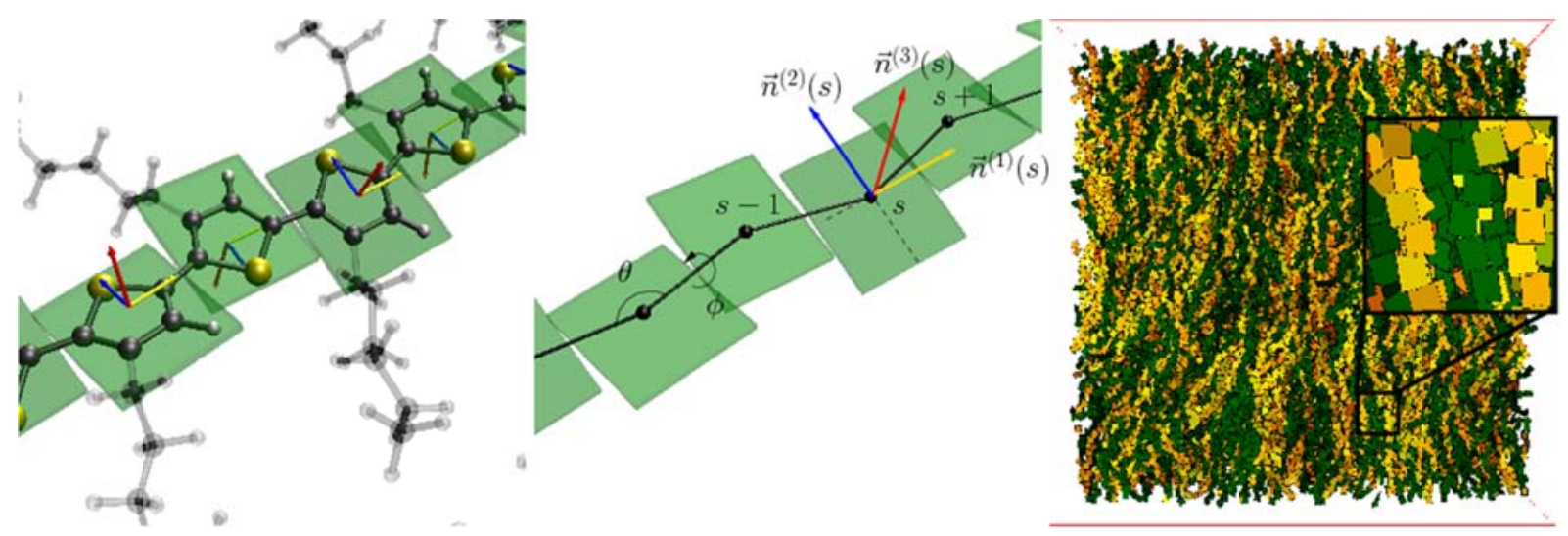

Figure 11. Atomistic and coarse-grained representation of a P3HT chain (left). Biaxial nematic alignment in a melt of P3HT chains (right). Adapted with permission from [109]. Copyright (2013) American Chemical Society.

The coarse-grained models with explicit beads for side chains are still too complex for simulations of dense systems and long chains. Further reducing the number of degrees of freedom, for instance, by removing coarse-grained side chains (see Figure 11), necessitates orientationally anisotropic interaction potentials, needed to account for the energetically favorable stacking of backbones. The symmetry of the mesophase (nematic, smectic, lamellar) can then be directly included into this anisotropic interaction potential. ${ }^{[110,111]}$. With 
these (soft) models, one can equilibrate morphologies as large as $50 \times 50 \times 50 \mathrm{~nm}^{3}$, containing in the order of $5 \times 10^{5}$ hexylthiophenes with up to 32 monomers per chain. ${ }^{[111]}$ With these morphologies at hand, some insights can be obtained on how the collective orientation of chains affects the energetic landscape for drift-diffusing charges. This has been done by splitting polymer segments into conjugated segments (using a simple criterion for the torsion angle ${ }^{[112]}$ ) and evaluating gas-phase ionization energies of these segments. Even this crude model predicted that isotropic melts consist of short conjugated segments with defects uniformly distributed along the chains. In the biaxial nematic case, the average segment length increased significantly, and the collective orientation of these segments led to a spatially-correlated energetic landscape, even without accounting for long-range Coulomb interactions. $^{[111]}$

Let us now discuss the semiconducting properties of P3HT. When choosing an appropriate model for charge transport in this polymer, we have to rely on the experimentally observed increase in mobility with increasing temperature. This is interpreted as a sign of temperatureactivated hopping transport. In other words, charges (charged states) are localized and charge transfer reactions that propagate the localized states are thermally activated. Localization is often interpreted in terms of conjugated segments, or part of a chain between the conjugationbreaking deviations of the backbone from planarity. ${ }^{[113]}$ This idea has been used to interpret spectroscopic data, but it is very difficult to apply to computational systems. First of all, it is not clear how one can rigorously define a sharp threshold separating the complete conjugation breaking from full conjugation between monomers. Moreover, there are many polymers (including P3HT) that form crystalline domains so extended that no conjugation breaks are found for tens of nanometers ${ }^{[114]}$ and the charge is certainly more localized than that, either by the disorder present in the semi-crystalline phase or by electron-phonon coupling ${ }^{[115,116]}$. To determine the charge localization there is no other alternative than computing the electronic wavefunction of a large model system, generated by classical simulation of polymers 
containing thousands of atoms. It is not practical to study them in a charged state using quantum-mechanical methods: A calculation of the electronic structure of such a large model with an electron less than the neutral state would only yield the ground state, while one is generally interested in the energy distribution and localization of many charged states. The excited state of the charged simulation box cannot be computed with modern computational methods and therefore one cannot even evaluate the nuclear relaxation (reorganization energy) for the hopping between two states. For these reasons all attempts to evaluate the wavefunction of a large model of polymers have focused on neutral systems and have interpreted the one-electron states (the orbitals) as the possible sites where the excess charge can be localized. ${ }^{[117,118]}$ Results are normally presented in terms of a density of states (DOS) and localization length, but it should be noticed that these two quantities are computed for the system with orbitals fully filled and empty above and below the band gap, respectively: they do not include effects of electron and nuclear polarization. For the one-electron states to be representative of the actual localization of the charge carrier the charge needs to be localized predominantly by the conformational disorder of the polymer and not by the electron-phonon coupling. If this coupling is particularly strong, the frontier orbitals may be delocalized over many monomers but, when an excess charge is added, the nuclear polarization will localize the charge, completely modifying the electronic wavefunction with respect to the neutral calculation. The localization of the wavefunction in P3HT is largely originating from disorder and not from electron-phonon coupling as there is a correlation between increased order of P3HT and increased mobility. ${ }^{[119,120,71]}$ Alternatively, one can be convinced that the conformational disorder is stronger than the electron-phonon coupling by comparing the polaron size of a perfectly ordered polymer chain with the localization of the orbitals computed from calculation of large realistic models of disordered chains. If the polaron size of the perfectly ordered system is much larger than the orbital in the disordered model it is acceptable to determine the charge localization from calculations that neglect the electron- 
phonon coupling as a first approximation. Calculations for P3HT support this approximation, ${ }^{[117,121]}$ but it should be noted that polaron sizes are strongly dependent on the DFT methodology. ${ }^{[122]}$

Under the conditions that the calculation of a ground state wavefunction of a polymer model yields information of the charge localization, it is still not trivial to carry out such calculations. It is not known in advance how large should a model of bulk polymer be to reproduce the DOS and localization length without the results being affected by finite-size effects. The available calculations with P3HT suggests that a model containing chains of 20 to 40 monomers displays electronic properties that do not depend appreciably on the chain length $^{[123]}$ (it should not be forgotten though that the morphology depends on the chain length until much larger molecular weight ${ }^{[124,111]}$ ). Such models will contain tens of thousands of atoms, a number still one order of magnitude larger than what is normally achievable by the current software specialized in linear scaling $a b$ initio calculations. Various partitioning schemes proposed for polymer systems are therefore very often complemented by further approximations that take into account the chemical structure of the investigated system. For lamellar systems such as P3HT and PBTTT it is an excellent approximation to neglect completely the electronic coupling between lamellae therefore describing a system that is essentially two-dimensional. ${ }^{[125,117]}$ The described methodology provides the DOS and a measure of the localization length of the states relevant for transport, that are those located at the edge of the valence (or conduction) band for holes (or electrons). For all polymers (amorphous and semi-crystalline), the states deep in the tail of the DOS are more localized and the localization length increases as states deeper in the band are considered, as predicted by simplified generic polymer models. ${ }^{[126]}$ The chemical description of these states in the tail is potentially very useful because it may suggest possible routes to increase charge mobility by reducing the number of trap states. Calculations suggest that hole trap states (high-energy occupied orbitals) are found in regions of $\mathrm{P} 3 \mathrm{HT}$ where the conjugated backbone is more 
planar than on average. ${ }^{[127,117]}$ The possibility of different degrees of planarity generates regions where the HOMO-LUMO gap is smaller, which coincide with the more planar segments of P3HT. Not surprisingly, when regioregularity defects are introduced, the trap states become localized far away from the regioregularity defect and, for this reason, the total number of trap states is not much affected by the regioregularity as found also experimentally. ${ }^{[117]}$ By adopting fast methods for the calculation of the electronic structure, it is possible to monitor the lifetime of these trap states by repeating the electronic structure calculation for different snapshots obtained via classical molecular dynamics. For P3HT the most stable trap states are stable for many tens of picoseconds, and thus it is possible to consider these trap states as static defects from the point of view of the moving charge carrier. ${ }^{[127]}$ Further analysis showed that these local deformations within the semi-crystalline domain are stabilized by gauche conformations in the side chain.

Once the one-electron states of a large system have been evaluated it is still very challenging to calculate hopping rates between these approximate states. One can use a perturbative expression assuming that these states are coupled by non-adiabatic coupling terms which can be evaluated explicitly ${ }^{[18]}$ or can be approximated using the overlap between the absolute value of the wavefunction. ${ }^{[127]}$ However, the proposed expression does not contain the effect of nuclear polarization in the presence of an additional charge (reorganization energy) and so it is strictly valid in the limit of vanishing reorganization energy, similar to Miller-Abrahams rates. ${ }^{[3]}$ Using this approach it was highlighted that it is possible to have reduced broadening of the DOS due to increased order, but still have low mobility because the coupling between states is reduced. ${ }^{[128,129]}$ An alternative, ad hoc approach relies on partitioning of polymer chains on conjugated segments and evaluating the charge transfer rate between these segments. Within this framework, the simplest rate expression, ${ }^{[130,144]}$ 
$k_{A \rightarrow \mathrm{B}}=\frac{2 \pi}{\hbar} \frac{\left|J_{\mathrm{AB}}\right|^{2}}{\sqrt{4 \pi \lambda k_{\mathrm{B}} T}} \exp \left[-\frac{\left(\Delta U_{\mathrm{AB}}-\lambda\right)^{2}}{4 \lambda k_{\mathrm{B}} T}\right]$, depends on three microscopic parameters: reorganization energy $\lambda$, electronic coupling $J_{\mathrm{AB}}$, and driving force $\Delta U_{\mathrm{AB}}=U_{\mathrm{A}}-U_{\mathrm{B}}$, all of which can be evaluated using quantum-chemical methods, classical polarizable force-fields, or quantum-classical hybrids as discussed in the following sections. Note that various generalizations of this expression to quantum-mechanical modes have been derived. ${ }^{[132-136]}$

For $\mathrm{P} 3 \mathrm{HT}$, the reorganization energy decreases to $0.1 \mathrm{eV}$ as of a chain length of 20 monomers, which is small compared to $0.2-0.4 \mathrm{eV}$ observed in many small-molecule based organic semiconductors also due to the better delocalization of the charge. Additionally, steric hindrance prevents conformational changes of the polymer chain upon charging if embedded in a $\pi$-stacked crystal. The resulting constraint on the backbone planarity lowers the reorganization energy.

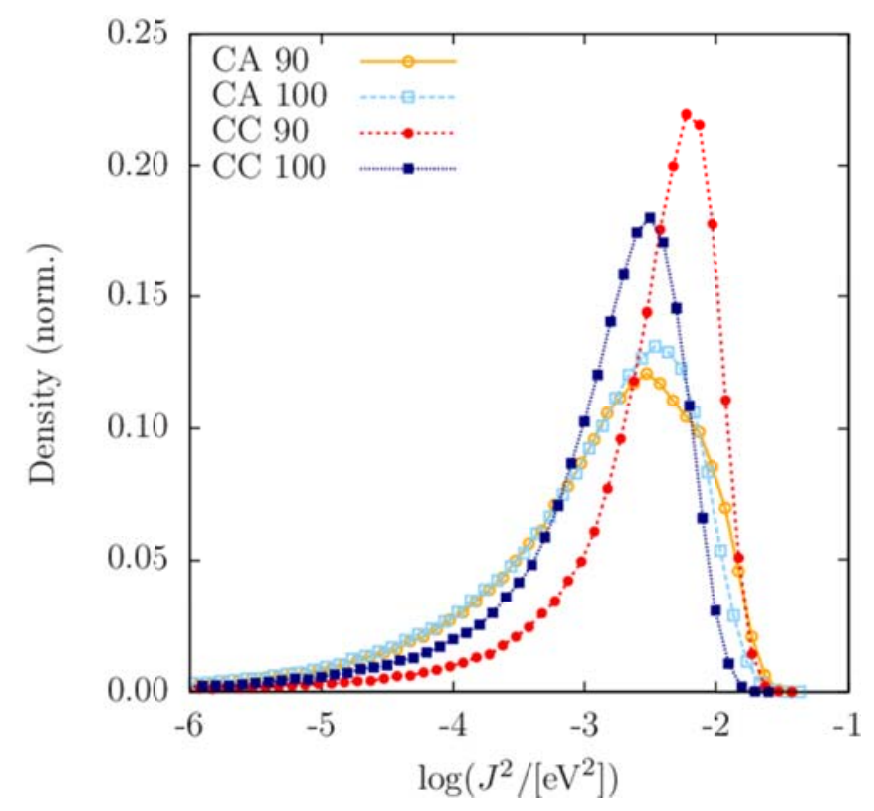

Figure 12. Distributions of squared electronic couplings for CC-100, CA-100, CC-90, CA-90. We use $\mathrm{C}(\mathrm{A})$ to denote crystalline (amorphous) packing, i.e. CA-100 corresponds to a system with a crystalline arrangement of backbones, amorphous packing of side-chains, and regioregularity of $100 \%$. Adapted with permission from Poelking et al, Macromolecules 46, 8941 (2013). Copyright (2013) American Chemical Society.

Let us now analyze the distribution of electronic couplings in P3HT crystals, which is shown in Figure 12. Unexpectedly, the $100 \%$ regioregular P3HT with crystalline side chains (CC- 
100) has (on average) lower electronic couplings than the corresponding $90 \%$ regioregular phase (CC-90). This effect is due to the different interlevel shift observed for the two regioregularities, since transfer integrals tend to be very sensitive to this structural mode. ${ }^{[136]}$ For two perfectly aligned chains the coupling element $\left|J_{\mathrm{AB}}\right|^{2}$ can vary between 0 and $10^{-2} \mathrm{eV}$ as the backbones are shifted with respect to each other along the polymer's long axis by one repeat unit, yielding a $\sin ^{2}$-type variation of $\left|J_{\mathrm{AB}}\right|^{2}$ with interlevel shift. Side-chain melting leads to a broadening of the distributions and a tail of very small couplings, down to $10^{-6} \mathrm{eV}$ (even though only nearest neighbors are present in the neighbor list). This would obviously result in rather small average mobility values. ${ }^{[137]}$ However, this conclusion is valid only if (one-dimensional) charge-carrier transport were to occur within a static snapshot of the system. In reality, both transfer integrals and site energies are time-dependent. In order to understand whether such a static picture can be used in the case of P3HT in what follows we compare the distributions of relaxation times of the electronic coupling elements and site energies to the distribution of escape times of a charge carrier.

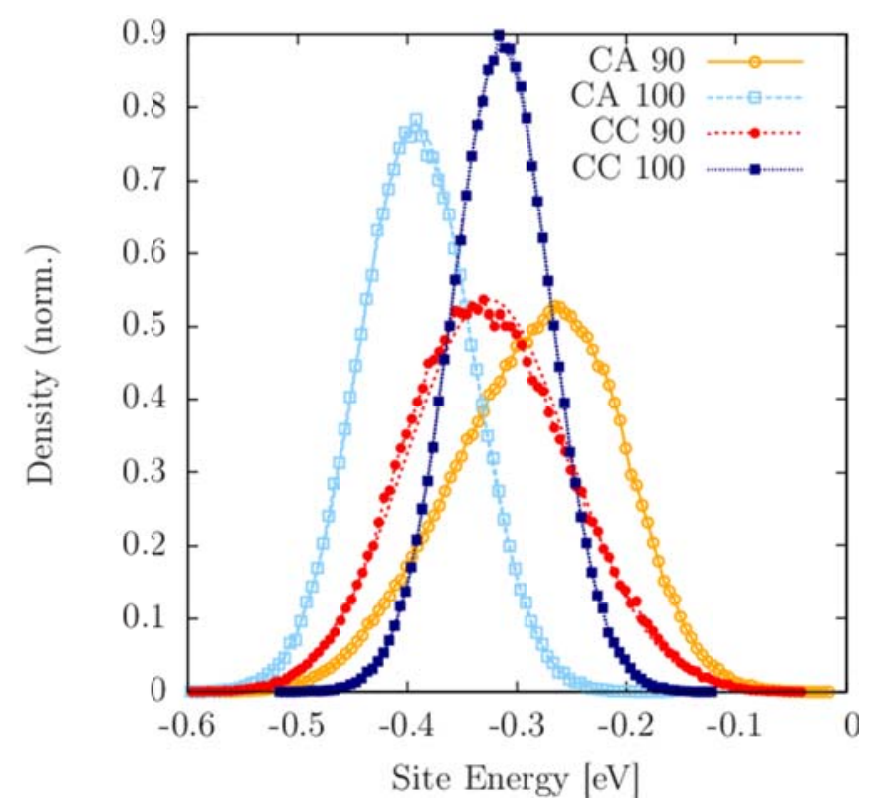

Figure 13. Densities of states together with Gaussians fitted to CC-100, CA-100, CC-90. Note the aberration from a Gaussian line shape found for CA-90, which results from slipping defects in the lamellar stack. We use $\mathrm{C}(\mathrm{A})$ to denote crystalline (amorphous) packing, that is, CA-100 corresponds to a system with a crystalline arrangement of backbones, amorphous packing of side-chains, and a regioregularity of 100\%. Adapted with permission from [94]. Copyright (2013) American Chemical Society. 
Another important ingredient of the charge transfer rate is the site energy difference, or the driving force $\Delta U_{\mathrm{AB}}$. It can be evaluated by expanding the molecular field and field response in terms of distributed multipoles and polarizabilities. Only a few studies have discussed the influence of this perturbation on the transport behavior of P3HT: systems of up $10^{4}$ thiophenes have been treated in this fashion, for example, in order to explore the density of states of P3HT in dependence on polymorph and regioregularity or at interfaces. ${ }^{[137,95]}$ In crystalline systems, P3HT backbones are fully conjugated and a charge can be assumed to delocalize over an entire oligomer. ${ }^{[117]}$ The internal contribution to the ionization potential does not change from segment to segment and the energetic disorder is mostly due to a locally varying electrostatic potential. The resulting distributions in hole site energies, that is, the differences between the energies of the system when a selected molecule is in the cationic or neutral state are shown in Figure 13, together with the fits to a Gaussian function. Here, both the width $\sigma$ and the mean $\langle U\rangle$ of the distribution depend on the side-chain packing and polymer regioregularity. As expected, 100\% regioregular P3HT always has narrower site energy distributions than the 90\% P3HT. Interestingly, the hole becomes less stable upon side-chain melting in the $100 \%$ regioregular P3HT (the distribution shifts to more negative numbers by $0.1 \mathrm{eV}$ ), while it is stabilized upon side chain melting in the $90 \%$ regioregular P3HT. One can attribute the changes in the energetic DOS to specific structural features. ${ }^{[95]}$ The width in the distributions is governed by regioregularity, with $\mathrm{CC}-100$ and $\mathrm{CA}-100$ having virtually identical widths of $\sigma=45 \mathrm{meV}$ and $51 \mathrm{meV}$, whereas CC-90, CA-90 are energetically more disordered with $\sigma=74 \mathrm{meV}$ and $75 \mathrm{meV}$, respectively. The magnitude of the disorder compares well with the width of the DOS as extracted from time-of-flight experiments, ${ }^{[68,41]}$ where values for $\sigma$ of $56 \mathrm{meV}$ and $71 \mathrm{meV}$, respectively, have been proposed from a fit of the field-dependence of the mobility as obtained within the Gaussian Disorder Model. ${ }^{[139]}$ Even though $\sigma$ 's did not vary significantly between $94 \%$ and $98 \%$ regioregular $\mathrm{P} 3 \mathrm{HT},{ }^{[41]}$ an increase in DOS width in the order of $30 \mathrm{meV}$ can already 
drastically impact charge mobility in the case of a one-dimensional connected hopping network, as can be assumed to appropriately reflect conditions in crystalline lamellae. On the level of chain ordering, the increase in energetic disorder can also be related to the increase in para-crystallinity along the $\pi$-stacking direction. ${ }^{\left[{ }^{95]}\right.}$ Considering that the hole-quadrupole interactions associated with thiophene dimers is the leading contribution to site energies, this origin for the increase in $\sigma$ is fully justified, and explains the similar energetic disorder computed for CC-100 and CA-100.

Shifts of the mean $\langle U\rangle$ of the site-energy distributions can be linked to the negative quadrupole moment of thiophene dimers: A hole localized on a polymer chain will be stabilized already on an electrostatic level due to the quadrupole moment of the neighboring chains, even without including polarization. This means that better geometrical overlap of the backbones leads to a larger stabilization of holes. During the transition from a staggered to a coplanar stacking, the reduction in tilt angle leads to enhanced hole-quadrupole interactions. These are responsible for the lower $\langle U\rangle$ in CA-100 compared to CC-100. For the systems of lower regioregularity, the transition in backbone stacking is less pronounced, since side-chain defects already lead to a slight planarization of the backbone, and hence lowered ionization potentials (compare the site-energy mean of CC-90 to CC-100). As the backbone stacking turns entirely coplanar, side-chain defects lead to a high degree of slipping-type paracrystallinity. This implies a weakening of the energetically favorable hole-quadrupole interaction, and therefore an increase in the mean of the site-energy distribution when comparing CC-90 to CA-90. In addition to this shift of the mean, slipping defects in CA-90 lead to a slight deviation from a Gaussian shape of the DOS. Summing up, the external contribution to the energetic density of states in P3HT is intimately connected to paracrystallinity along the $\pi$-stacking direction, with energetic disorder $\sigma$ linearly related to the amplitude of backbone-backbone distance fluctuations, and the mean of the backbonebackbone distance distribution analogously related to the average site energy $\langle U\rangle$. 


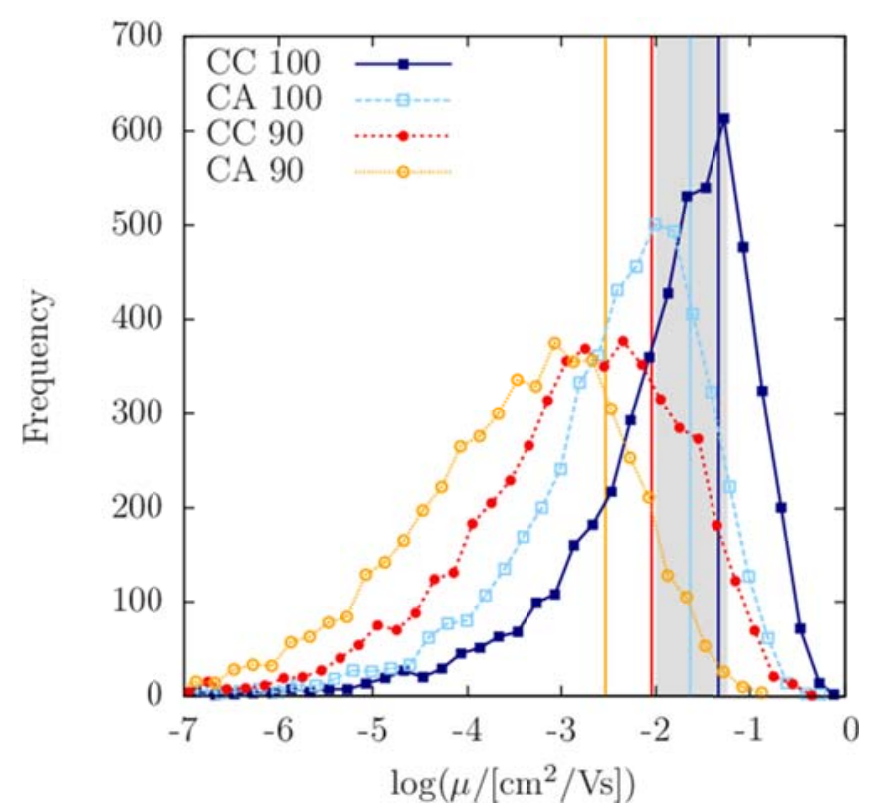

Figure 14. Distributions of lamellar mobilities for CC-100, CA-100, CC-90, and CA-90. Vertical lines indicate averages. The gray bar includes the range of mobilities measured in P3HT nanofibers. ${ }^{[72,145,74]}$ We use C(A) to denote crystalline (amorphous) packing, that is, CA-100 corresponds to a system with a crystalline arrangement of backbones, amorphous packing of side-chains, and regioregularity of 100\%. Adapted with permission from [94]. Copyright (2013) American Chemical Society.

With electronic couplings and driving forces at hand, one can evaluate charge transfer rates, solve the resulting master equation for occupation probabilities of these localized states and extract information about charge distribution, currents, and eventually mobility, all as a function of temperature, external field, charge density, and, importantly, morphology. Studies of different levels of complexity have been performed to study hole transport along the $\pi$ stacking direction of P3HT lamellae. ${ }^{[95,138,140]}$ Since the transport has a one-dimensional character, it can already be anticipated that a broad and static distribution of electronic couplings limits charge mobility along lamellae. ${ }^{[131,136,141-146]}$ This is illustrated in Figure 14: mobility values, evaluated for 5000 lamellae, each consisting of 40 stacked chains, are broadly distributed, with small mobilities as low as $10^{-7} \mathrm{~cm}^{2} / \mathrm{Vs}$.

It is important to relate the distribution of mobilities to those of electronic couplings and site energies. It has been found by comparing materials of different regioregularity that the associated mobility distributions are fundamentally different from what one would expect solely on the grounds of electronic couplings, which are shown in Figure 12. The distribution 
of transfer integrals is determined by the polymorph at hand (I' or I) and is not sensitive to a small decrease in regioregularity, whereas energetic disorder is governed by regioregularity defects and is polymorph-independent. Aiming for high mobilities, one should hence prefer high regioregularity over medium regioregularity due to the smaller energetic disorder and P3HT form I' over P3HT form I due to higher electronic couplings. The mobility implicitly depends on both quantities: the average mobility decreases from CC-100 to CA-100 to CC-90 to CA-90. ${ }^{[94]}$ These averages are indicated by vertical bars in Figure 13 . In the case of $100 \%$ regioregular P3HT, simulation results are in excellent agreement with field-effect mobilities in P3HT nanofibers (devoid of grain boundaries) extracted from experimental transistor I-V curves on P3HT nanofibers ${ }^{[74,147,76]}$ The range of experimental values $(\mu=0.01-$ $0.06 \mathrm{~cm}^{2} / \mathrm{Vs}$ ) obtained for different solvent and processing conditions is shown as the gray bar in Figure 14.

The effect of regioregularity on charge transport has been studied experimentally in the context of time-of-flight experiments, ${ }^{[41]}$ where a reduction in regioregularity by $5 \%$ led to a decrease in mobility by a factor of five. In simulations, a reduction in regioregularity by $10 \%$ translates into a factor of ten decrease in mobility, indicating that the decrease in mobility is due to intradomain instead of interdomain transport. Hence, the regioregularity effect is exclusively due to increased energetic disorder. ${ }^{[95]}$ Additionally, the intramolecular contribution to the density of states was found to have a negligible effect on localization length and hence transport in the high-regioregularity regime. ${ }^{[117]}$ Thus, the higher mobility in the more regioregular material is entirely attributable to a narrowing of the density of states that results from increased order in hole-quadrupole interaction distances.

In the hopping picture, understanding the factors that limit charge-carrier mobility in polymers not only demands knowledge of the distribution of transport parameters, but also their time dependence. The latter is an important hint as to whether or not the hopping picture is justified to begin with, since there is in fact no theoretical technique available that can 
predict the regime of charge transport for a given material system. In P3HT, the relaxation of the electronic coupling elements and site energies occurs on similar time scales in spite of their dissimilar physical origins: Site energies are related to long-range electrostatic interactions where averaging occurs over a large number of nearest neighbors and leads to spatial correlations. ${ }^{[95]}$ On the other hand, the electronic coupling elements (to a first approximation) only depend on the geometries of pairs of molecules, which results in increased sensitivity to thermal motions of the internal degrees of freedom. The reason for similar time scales is the chemical structure of P3HT: Every thiophene unit is linked to an alkyl side chain with slow dynamics both in the crystalline and amorphous phases. This overdamps the backbone dynamics, in particular torsional motions of thiophene units, and results in slow variations of electronic couplings. Interestingly, for the similar conjugated polymer PBTTT, where the thienothiophene unit is not linked to a side chain (implying a lower side-chain density and better crystallinity), the significantly faster dynamics of electronic couplings can boost the charge-carrier mobility. ${ }^{[96]}$ For P3HT, even the slowest escape times for holes do not extend into the decorrelation regime. Charge-carrier dynamics is therefore limited by the static disorder of electronic couplings and site energies, since their relaxation times exceed typical time scales of hopping transport. Hence, it is possible to resort to a single charge-transfer rate to describe transport without time-averaging of electronic couplings of a pair of molecules, as it was needed in columnar discotic liquid crystals ${ }^{[148]}$ or PBTTT. ${ }^{[95]}$

\section{Conclusions and Outlook}

The charge transport and photogeneration in regioregular poly(3hexylthiophene):methanofullerene (P3HT:PCBM) solar cells is reviewed. A ten-fold increase in power conversion efficiency was obtained by simply annealing the devices at a temperature above $110{ }^{\circ} \mathrm{C}$ for $4 \mathrm{~min}$. The most important factor in obtaining these 
efficiencies was found to be the enhancement in hole mobility in the P3HT phase of the blend by more than three orders of magnitude, relative to the as-cast device. For the devices as-cast, or annealed at a temperature lower than optimal $\left(<110{ }^{\circ} \mathrm{C}\right)$, the difference in electron and hole transport in the blend is too large and the photocurrent is strongly limited by the build-up of space-charge. Consequently, the devices are hindered by the fundamental electrostatic limit and the fill factor of the cells cannot exceed $42 \%$. At optimum annealing temperature (above $110{ }^{\circ} \mathrm{C}$ ), the difference in electron and hole mobility is reduced and the space-charge no longer limits the performance under normal operation conditions leading to fill factors as high as $70 \%$. Furthermore, at short-circuit conditions the dissociation efficiency of bound electron-hole pairs at the donor/acceptor interface is close to $90 \%$, which explains the large quantum efficiencies measured in P3HT:PCBM blends. These results are valuable for the design of new materials and further improve the performance of organic photovoltaic devices. On the theoretical side, an adequate understanding of highly crystalline and completely amorphous systems has been achieved. However, for partially ordered systems substantial method development is still required: Imperative for predicting large-scale morphologies are accurate polarizable force-fields and computationally efficient coarsegrained models. These models should be capable of describing backbone crystallization and allow for reintroduction of atomistic details. Essential for charge transport are extensions of large-scale first-principles methods to charged states. Challenging is the incorporation of polaronic effects and a unified description of charge transfer along a single chain and between conjugated segments located on different chains.

Acknowledgements: F. Laquai thanks the Max Planck Society for funding the Max Planck Research Group. R. Mauer acknowledges funding from the Max Planck Graduate Center with the University of Mainz (MPGC). This work was partly supported by the DFG program IRTG 
1404, DFG grant SPP 1355, and BMBF grants MEDOS (FKZ 03EK3503B) and MESOMERIE (FKZ 13N10723).

Received: Month XX, XXXX; Revised: Month XX, XXXX; Published online:

Keywords: poly(3-hexylthiophene), charge recombination, charge transport, DFT calculations, organic solar cells

[1] E. M. Conwell, Phys. Rev. 1956, 103, 51-61.

[2] N. F. Mott, Can. J. Phys. 1956, 34, 1356-1368.

[3] A. Miller and E. Abrahams, Phys. Rev. 1960, 120, 745 (1960).

[4] M. Van der Auweraer, F. C. De Schryver, P. M. Borsenberger, and H. Bässler, Adv. Mater. 1994, 6, 199-213.

[5] P. W. M. Blom, M. J. M. de Jong, and M. G. van Munster, Phys. Rev. B 1997, 55, R656R659.

[6] J. Frenkel, Phys. Rev. 1938, 54, 647-648.

[7] H. Bässler, Phys. Stat. Sol. (b) 1993, 175, 15-56.

[8] Y. N. Gartstein and E. M. Conwell, Chem. Phys. Lett. 1995, 245, 351-358.

[9] D. H. Dunlap, P. E. Parris, and V. M. Kenkre, Phys. Rev. Lett. 1996, 77, 542.

[10] S. V. Novikov, D. H. Dunlap, V. M. Kenkre, P. E. Parris, and A. V. Vannikov, Phys.

Rev. Lett. 1998, 81, 4472.

[11] C. Tanase, E. J. Meijer, P. W. M. Blom, and D. M. de Leeuw, Org. Electron. 2003 4, 3337.

[12] G. Horowitz, M. E. Hajlaoui, and R. Hajlaoui, J. Appl. Phys. 2000, 87, 4456-4463.

[13] A. R. Brown, C. P. Jarrett, D. M. de Leeuw, and M. Matters, Synth. Met. 1997, 88, 37-55.

[14] G. Horowitz, R. Hajlaoui, D. Fichou, and A. El Kassmi, J. Appl. Phys. 1999, 85, 32023206.

[15] C. D. Dimitrakopoulos, S. Purushothaman, J. Kymissis, A. Callegari, and J. M. Shaw, Science 1999, 283, 822-824.

[16] M. C. J. M. Vissenberg and M. Matters, Phys. Rev. B 1998, 57, 12964.

[17] C. Tanase, E. J. Meijer, P. W. M. Blom, and D. M. de Leeuw, Phys. Rev. Lett. 2003, 91, 216601.

[18] C. Tanase, P. W. M. Blom, and D. M. de Leeuw, Phys. Rev. B 2004, 70, 193202.

[19] W. F. Pasveer, J. Cottaar, C. Tanase, R. Coehoorn, P. A. Bobbert, P. W. M. Blom, D. M. de Leeuw, and M. A. J. Michels, Phys. Rev. Lett. 2005, 94, 206601.

[20] O. Tal, Y. Rosenwaks, Y. Preezant, N. Tessler, C. K. Chan, and A. Kahn, Phys. Rev. Lett. 2005, 95, 256405.

[21] N. Vukmirović and L.-W. Wang, J. Phys. Chem. B 2011, 115, 1792-1797.

[22] V.D. Mihailetchi, H. Xie, B. de Boer, L.J.A Koster, and P.W.M. Blom, Adv. Funct. Mat. 2006, 16, 699 .

[23] The photocurrent $\left(J_{\mathrm{ph}} ; J_{\mathrm{ph}}=J_{\mathrm{L}}-J_{\mathrm{D}}\right)$ is the measured current under illumination $\left(J_{\mathrm{L}}\right)$ corrected for the dark current $\left(J_{\mathrm{D}}\right)$, whereas $V_{0}$ is the compensation voltage. $V_{0}$ is defined as the voltage at which $J_{\text {ph }}$ is zero. 
[24] F. Padinger, R. S. Rittberger, N. S. Sariciftci, Adv. Funct. Mater. 2003, 13, 85.

[25] D. Chirvase, J. Parisi, J. C. Hummelen, V. Dyakonov, Nanotechn. 2004, 15, 1317.

[26]Y. Kim, S. A. Choulis, J. Nelson, D. D. C. Bradley, S. Cook, J. R. Durrant, Appl.

Phys. Lett. 2005, 86, 063502.

[27] X. Yang, J. Loos, S. C. Veenstra, W. J. H. Verhees, M. M. Wienk, J. M. Kroon,M. A. J. Michels, R. A. J. Janssen, Nano Lett., 2005, 5, 579.

[28] V. D. Mihailetchi, L. J. A. Koster, P. W. M. Blom, C. Melzer, B. de Boer, J. K. J. van Duren, R. A. J. Janssen, Adv. Funct. Mater. 2005, 15, 795.

[29] L. J. A. Koster, E. C. P. Smits, V. D. Mihailetchi, P. W. M. Blom, Phys. Rev. B 2005, $72,085205$.

[30]V. D. Mihailetchi, L. J. Koster, J. C. Hummelen, P. W. Blom, Phys. Rev. Lett. 2004, 93, 216601.

[31] V. D. Mihailetchi, J. Wildeman, P. W. M. Blom, Phys. Rev. Lett. 2005, 94, 126602.

[32] H. Sirringhaus, P. J. Brown, R. H. Friend, M. M. Nielsen, K. Bechgaard, B. M. W.

Langeveld-Voss, A. J. H. Spiering, R. A. J. Janssen, E. W. Meijer, P. Herwig, D. M. de

Leeuw, Nature, 1999, 401, 685.

[33] R. J. Kline, M. D. McGehee, E. N. Kadnikova, J. S. Liu, J. M. J. Frechet, $A d v$.

Mater. 2003, 15, 1519.

[34] Y. Kim, S. Cook, S. A. Choulis, J. Nelson, J. R. Durrant, D. D. C. Bradley, Chem. Mater. 2004, 16, 4812.

[35] C. Goh, R. J. Kline, M. D. McGehee, E N. Kadnikova, J. M. J. Fréchet, Appl. Phys. Lett. 2005, 86, 122110.

[36] H. Sirringhaus, P. J. Brown, R. H. Friend, M. M. Nielsen, K. Bechgaard, B. M. W. Langeveld-Voss, A. J. H. Spiering, R. A. J. Janssen, E. W. Meijer, P. Herwig D. M. de Leeuw, Nature 1999, 401, 685.

[37] C. Melzer, E. J. Koop, V. D. Mihailetchi, P. W. M. Blom, Adv. Funct. Mater. 2004, 14,865 .

[38] Yang, K.; Wang, Y.; Jain, A.; Samulson, L.; Kumar, J. J. Macromol. Sci. Part A-Pure Appl. Chem. 2007, 44, 1261.

[39] J.S. Huang, G. Li, Y. Yang, Appl. Phys. Lett. 2005, 87.

[40] A. Baumann, J. Lorrmann, C. Deibel, V. Dyakonov, Appl. Phys. Lett. 2008, 93.

[41] R. Mauer, M. Kastler, F. Laquai, F. Adv. Funct. Mater. 2010, 20, 2085.

[42] A. Zen, J. Pflaum, S. Hirschmann, W. Zhuang, F. Jaiser, U. Asawapirom, J.P. Rabe, U. Scherf, D. Neher, Adv. Funct. Mater. 2004, 14, 757.

[43] F. Laquai, G. Wegner, C. Im, H. Bässler, S. Heun, J. Appl. Phys. 2006, 99.

[44] D. E. Markov, C. Tanase, P. W.M. Blom, J. Wildeman, Phys. Rev. B. 72, 045217 (2005).

[45] S.R. Cowan, R.A. Street, S.N. Cho, A.J. Heeger, Phys. Rev. B 2011, 83.

[46] A. Hadipour, B. de Boer, J. Wildeman, F. B. Kooistra, J. C. Hummelen, M. G. R.

Turbiez, M. M. Wienk, R. A. J. Janssen, P. W. M. Blom, Adv. Funct. Mat. 16, 1897 (2006).

[47] M. M. Mandoc, W. Veurman, J. Sweelssen, M. M. Koetse, P.W.M. Blom, Appl. Phys.

Lett. 91, 073518 (2007).

[48] G.F. Burkhard, E.T. Hoke, M.D. McGehee, Adv. Mater. 2010, 22, 3293.

[49] J.D. Kotlarski, P.W.M. Blom, M. Lenes, L.J.A. Koster, and L. Slooff,

J. Appl. Phys. 103, 084502 (2008)

[50] R.A. Marsh, J.M. Hodgkiss, S. Albert-Seifried, R.H. Friend, Nano Lett. 2010, 10, 923.

[51] I.A. Howard, R. Mauer, M. Meister, F. Laquai, J. Am. Chem. Soc. 2010, 132, 14866.

[52] S. Gelinas, A. Rao, A. Kumar, S.L. Smith, A.W. Chin, J. Clark, T.S. van der Poll, G.C.

Bazan, R.H. Friend, Science 2014, 343, 512.

[53] F.C. Jamieson, E.B. Domingo, T. McCarthy-Ward, M. Heeney, N. Stingelin, J.R. Durrant, Chem. Sci. 2012, 3, 485.

[54] R. Mauer, I.A. Howard, F. Laquai, J. Phys. Chem. Lett. 2010, 1, 3500. 
[55] J. Kniepert, M. Schubert, J.C. Blakesley, D. Neher, J. Phys. Chem. Lett. 2011, 2, 700.

[56] G. Juska, K. Genevicius, N. Nekrasas, G. Sliauzys, R. Osterbacka, R. Appl. Phys. Lett. 2009, 95.

[57] C.G. Shuttle, B. O'Regan, A.M. Ballantyne, J. Nelson, D.D.C. Bradley, J. de Mello, J.R. Durrant, Appl. Phys. Lett. 2008, 92.

[58] F. Etzold, I.A. Howard, R. Mauer, M. Meister, T.D. Kim, K.S. Lee, N.S. Baek, F. Laquai, J. Am. Chem. Soc. 2011, 133, 9469.

[59] C.G. Shuttle, R. Hamilton, J. Nelson, B.C. O'Regan, J.R. Durrant, Adv. Funct. Mater. 2010, 20, 698.

[60] T.M. Clarke, F.C. Jamieson, J.R. Durrant, J. Phys. Chem. C 2009, 113, 20934.

[61] L.J.A. Koster, M. Kemerink, M.M. Wienk, K. Maturova, R.A.J. Janssen, Adv. Mater. 2011, 23, 1670.

[62] C. Deibel, A. Wagenpfahl, V. Dyakonov, Phys. Rev. B 2009, 80.

[63] M. Soldera, K. Taretto, T. Kirchartz, Phys. Status Solidi A-Appl. Mat. 2012, 209, 207.

[64] Y. Kim, S. Cook, S.M. Tuladhar, S.A. Choulis, J. Nelson, J.R. Durrant, D.D.C. Bradley, M. Giles, I. McCulloch, C.S Ha, M. Ree, Nat. Mater. 2006, 5, 197.

[65] B.A. Collins, J.R Tumbleston, H. Ade, J. Phys. Chem. Lett. 2011, 2, 3135.

[66] R. Mauer, I.A. Howard, F. Laquai, J. Phys. Chem. Lett. 2011, 2 (14), 1736-1741.

[67] R.D. McCullough, and R. D. Lowe, Chem. Comm. 1992, (1), 70.

[68] A.M. Ballantyne, L. Chen, J. Dane, T. Hammant, F. M. Braun, M. Heeney, W. Duffy, I. McCulloch, D. D. C. Bradley, and J. Nelson, Adv. Funct. Mater. 2008, 18 (16), 2373-2380.

[69] A. Tsumura, H. Koezuka, and T. Ando, Appl. Phys. Lett. 1986, 49 (18), 1210.

[70] Z. Bao, A. Dodabalapur, and A. J. Lovinger, Appl. Phys. Lett. 1996, 69 (26), 4108.

[71] J.-F. Chang, J. Clark, N. Zhao, H. Sirringhaus, D. W. Breiby, J. W. Andreasen, M. M. Nielsen, M. Giles, M. Heeney, and I. McCulloch, Phys. Rev. B 2006, 74 (11), 115318.

[72] R.J. Kline, M.D. McGehee, E. N. Kadnikova, J. Liu, J. M. J. Fréchet, and M. F. Toney, Macromolecules 2005, 38 (8), 3312.

[73] A. Zen, J. Pflaum, S. Hirschmann, W. Zhuang, F. Jaiser, U. Asawapirom, J. P. Rabe, U. Scherf, and D. Neher, Adv. Funct. Mater. 2004, 14 (8), 757-764.

[74] J.-C. Bolsee, W. D. Oosterbaan, L. Lutsen, D. Vanderzande, and J. Manca, Organic Electronics 2011, 12 (12), 2084.

[75] J.A. Merlo, and C. D. Frisbie, J. Polym. Sci. Part B Polym. Phys. 2003, 41 (21), 26742680.

[76] T. Shimomura, T. Takahashi, Y. Ichimura, S. Nakagawa, K. Noguchi, S. Heike, and T. Hashizume, Phys. Rev. B 2011, 83 (11), 115314.

[77] G. Raos, A. Famulari, and V. Marcon, Chem. Phys. Lett. 2003, 379 (3-4), 364.

[78] T.J. Prosa, M. J. Winokur, and R. D. McCullough, Macromol. 1996, 29 (10), 3654.

[79] M. Brinkmann, J. Polym. Sci. Part B Polym. Phys. 2011, 49 (17), 1218.

[80] Z. Wu, A. Petzold, T. Henze, T. Thurn-Albrecht, R. H. Lohwasser, M. Sommer, and M. Thelakkat, Macromol. 2010, 43 (10), 4646.

[81] D. Dudenko, A. Kiersnowski, J. Shu, W. Pisula, D. Sebastiani, H. W. Spiess, and M. R. Hansen, Angew. Chem. Int. Edit. 2012, 51 (44), 11068.

[82] Y. Yuan, J. Zhang, J. Sun, J. Hu, T. Zhang, and Y. Duan, Macromol. 2011, 44 (23), 9341.

[83] S.B. Darling, and M. Sternberg, J. Phys. Chem. B 2009, 113 (18), 6215, PMID: 19290596.

[84] C. Marchiori, and M. Koehler, Synth. Met. 2010, 160 (7-8), 643.

[85] R.S. Bhatta, Y. Y. Yimer, M. Tsige, and D. S. Perry, Comput. Theoret. Chem. 2012, 995, 36.

[86] S. Tsuzuki, K. Honda, and R. Azumi, J. Am. Chem. Soc. 2002, 124 (41), 12200.

[87] R.S. Bhatta, and D. S. Perry, Comput. Theoret. Chem. 2013, 1008, 90. 
[88] A. Baggioli, and A. Famulari, Phys. Chem. Chem. Phys. 2014, 16, 3983.

[89] R. Colle, G. Grosso, A. Ronzani, and C. M. Zicovich-Wilson, phys. stat. sol. (b) 2011, 248 (6), 1360-1368.

[90] A. Famulari, G. Raos, A. Baggioli, M. Casalegno, R. Po, and S. V. Meille, J. Phys. Chem. B 2012, 116 (49), 14504.

[91] A Maillard, and A. Rochefort, Phys. Rev. B 2009, 79 (11).

[92] W. Xie, Y. Y. Sun, S. B. Zhang, and J. E. Northrup, Phys. Rev. B 2011, 83 (18), 184117.

[93] S. Dag, and L.-W. Wang, J. Phys. Chem. B 2010, 114 (18), 5997.

[94] V. Marcon, and G. Raos, J. Phys. Chem. B 2004, 108 (46), 18053.

[95] C. Poelking, and D. Andrienko, Macromol. 2013, 46 (22), 8941.

[96] R.S. Bhatta, Y. Y. Yimer, D. S. Perry, and M. Tsige, J. Phys. Chem. B 2013, 117 (34), 10035.

[97] T.T. To, and S. Adams, Nanosci. Nanotech. Lett. 2012, 4 (7), 703.

[98] C. Melis, L. Colombo, and A. Mattoni, J. Phys. Chem. C 2011, 115 (2), 576.

[99] O. Alexiadis, and V. G. Mavrantzas, Macromol. 2013, 46 (6), 2450.

[100] D. Curco, and C. Aleman, J. Comput. Chem. 2007, 28 (10), 1743-1749.

[101] G. Zhang, Y. Pei, J. Ma, K. Yin, and C.-L. Chen, J. Phys. Chem. B 2004, 108 (22),

[102] K. Do, D. M. Huang, R. Faller, and A. J. Moulé, Phys. Chem. Chem. Phys. 2010, 12 (44), 14735.

[103] D.M. Huang, R. Faller, K. Do, and A. J. Moulé, J. Chem. Theory. Comput. 2010, 6 (2), 526.

[104] Y.Y. Yimer, A. Dhinojwala, and M. Tsige, J. Chem. Phys. 2012, 137 (4), 044703.

[105] S. Reddy, and V. K. Kuppa, Synth. Met. 2012, 162 (23), 2117.

[106] G. D’Avino, S. Mothy, L. Muccioli, C. Zannoni, L. Wang, J. Cornil, D. Beljonne, and

F. Castet, J. Phys. Chem. C 2013, 117 (25), 12981.

[107] C.-K. Lee, C.-W. Pao, and C.-W. Chu, Energy Environ. Sci. 2011, 4 (10), 4124.

[108] E. Jankowski, H. S. Marsh, and A. Jayaraman, Macromol. 2013, 46 (14), 5775.

[109] K.N. Schwarz, T. W. Kee, and D. M. Huang, Nanoscale 2013, 5 (5), 2017.

[110] K.C. Daoulas, V. Rühle, and K. Kremer, J. Phys. Condens. Mat. 2012, 24 (28), 284121.

[111] P. Gemünden, C. Poelking, K. Kremer, D. Andrienko, and K. C. Daoulas, Macromol. 2013, 46 (14), 5762.

[112] V. Rühle, J. Kirkpatrick, and D. Andrienko, J. Chem. Phys. 2010, 132 (13), 134103.

[113] E. Collini, and G. D. Scholes, Science 2009, 323 (5912), 369, PMID: 19150843.

[114] A. Salleo, R. J. Kline, D. M. DeLongchamp, and M. L. Chabinyc, Adv. Mater. 2010, 22 (34), 3812-3838.

[115] D. Beljonne, J. Cornil, H. Sirringhaus, P. J. Brown, M. Shkunov, R. H. Friend, and J.-L. Brédas, Adv. Funct. Mater. 2001, 11 (3), 229-234.

[116] P.J. Brown, H. Sirringhaus, M. Harrison, M. Shkunov, and R. H. Friend, Phys. Rev. B 2001, 63 (12), 125204.

[117] D.P. McMahon, D. L. Cheung, L. Goris, J. Dacuna, A. Salleo, and A. Troisi, J. Phys. Chem. C 2011, 115 (39), 19386.

[118] N. Vukmirovic, and L.-W. Wang, J. Phys. Chem. B 2011, 115 (8), 1792.

[119] Van der Auweraer, M., F. C. De Schryver, P. M. Borsenberger, and H. Bässler, Adv. Mater. 1994, 6 (3), 199-213.

[120] E.H. Magin, and P. M. Borsenberger, J. Appl. Phys. 1993, 73 (2), 787.

[121] S.S. Zade, N. Zamoshchik, and M. Bendikov, Acc. Chem. Res. 2011, 44 (1), 14.

[122] I.H. Nayyar, E. R. Batista, S. Tretiak, A. Saxena, D. L. Smith, and R. L. Martin, J. Chem. Theory. Comput. 2013, 9 (2), 1144.

[123] T. Qin, and A. Troisi, J. Am. Chem. Soc. 2013, 135 (30), 11247.

[124] S. Shaked, S. Tal, Y. Roichman, A. Razin, S. Xiao, Y. Eichen, and N. Tessler, Adv. Mater. 2003, 15 (11), 913-916. 
[125] J. Liu, Y. Sun, X. Gao, R. Xing, L. Zheng, S. Wu, Y. Geng, and Y. Han, Langmuir 2011, 27 (7), 4212.

[126] R. Noriega, J. Rivnay, K. Vandewal, F. P. V. Koch, N. Stingelin, P. Smith, M. F. Toney, and A. Salleo, Nat. Mater. 2013, 12 (11), 1038.

[127] D.L. Cheung, D. P. McMahon, and A. Troisi, J. Am. Chem. Soc. 2009, 131 (31), 11179.

[128] N. Vukmirovic, and L.-W. Wang, Appl. Phys. Lett. 2010, 97 (4), 043305.

[129] N. Vukmirovic, Phys. Chem. Chem. Phys. 2013, 15 (10), 3543.

[130] R.A. Marcus, Rev. Mod. Phys. 1993, 65 (3), 599.

[131] F. May, V. Marcon, M. R. Hansen, F. Grozema, and D. Andrienko, J. Mater. Chem. 2011, 21 (26), 9538.

[132] K. Asadi, A. J. Kronemeijer, T. Cramer, L. Jan Anton Koster, P. W. M. Blom, and D. M. de Leeuw, Nat. Comm. 2013, 4, 1710.

[133] M. P. A. Fisher, and A. T. Dorsey, Phys. Rev. Lett. 1985, 54 (15), 1609.

[134] H. Grabert, and U. Weiss, Phys. Rev. Lett. 1985, 54 (15), 1605.

[135] V. Rühle, A. Lukyanov, F. May, M. Schrader, T. Vehoff, J. Kirkpatrick, B. Baumeier, and D. Andrienko, J. Chem. Theory. Comput. 2011, 7 (10), 3335.

[136] Y. Fogel, L. Zhi, A. Rouhanipour, D. Andrienko, H. J. Räder, and K. Müllen, Macromolecules 2009, 42 (18), 6878.

[137] H. Scher, S. Alexander, and E. W. Montroll, Proc. Natl. Acad. Sci. USA 1980, 77 (7), 3758.

[138] C. Poelking, V. Ivanov, K. Kremer, C. Risko, J.-L. Brédas, D. Andrienko, and C. Eunkyung, J. Phys. Chem. C 2013, 117 (4), 1633.

[139] H. Bässler, Phys. Stat. Sol. B 1993, 175 (1), 15.

[140] Y.-K. Lan, C. H. Yang, and H.-C. Yang, Poly. Inter. 2010, 59 (1), 16.

[141] D. Andrienko, J. Kirkpatrick, V. Marcon, J. Nelson, and K. Kremer, Phys. Stat. Sol. B 2008, 245 (5), 830.

[142] J. Kirkpatrick, V. Marcon, J. Nelson, K. Kremer, and D. Andrienko, Phys. Rev. Lett. 2007, 98 (22), 227402.

[143] V. Marcon, J. Kirkpatrick, W. Pisula, and D. Andrienko, Phys. Stat. Sol. B 2008, 245 (5), 820-824.

[144] V. May, and O. Kuhn (2011), Charge and Energy Transfer Dynamics in Molecular Systems, 3rd ed. (Wiley-VCH).

[145] J. Nelson, J. J. Kwiatkowski, J. Kirkpatrick, and J. M. Frost, Acc. Chem. Res. 2009, 42 (11), 1768.

[146] a) M. Schrader, R. Fitzner, M. Hein, C. Elschner, B. Baumeier, K. Leo, M. Riede, P. Bäuerle, and D. Andrienko, J. Am. Chem. Soc. 2012, 134 (13), 6052. b) M. Schrader, C. Körner, C. Elschner, and D. Andrienko, J. Mater. Chem. 2012, 22 (41), 22258.

[147] M. Mas-Torrent, D. d. Boer, M. Durkut, P. Hadley, and A. P. H. J. Schenning, Nanotech. 2004, 15 (4), 265.

[148] Y. Olivier, L. Muccioli, V. Lemaur, Y. H. Geerts, C. Zannoni, and J. Cornil, J. Phys. Chem. B 2009, 113 (43), 14102. 